\title{
L'extraction du granite rosé de l'île Callot et son emploi dans le pays
} de Morlaix (Finistère)

Louis Chauris

\section{Citer ce document / Cite this document :}

Chauris Louis. L'extraction du granite rosé de l'île Callot et son emploi dans le pays de Morlaix (Finistère). In: Annales de Bretagne et des pays de l'Ouest. Tome 102, numéro 1, 1995. pp. 7-34;

doi : https://doi.org/10.3406/abpo.1995.3803

https://www.persee.fr/doc/abpo_0399-0826_1995_num_102_1_3803

Fichier pdf généré le 04/04/2018 


\title{
Résumé
}

La reconnaissance de très nombreux anciens sites d'extraction du granité de Callot sur les estrans de l'île et les récifs voisins en baie de Morlaix, l'examen systématique des différents types de constructions (édifices religieux, châteaux et manoirs, habitat des cités et des campagnes...) dans toute la région morlaisienne, et les données d'archives (peu nombreuses), révèlent pour la première fois l'importance prise dans le passé par cette belle pierre rosée, à présent bien oubliée. La répartition spatiale des constructions souligne le rôle majeur joué par le transport maritime (baie de Morlaix prolongée par les rias du Dossen et de Penzé) de ce granité, lui assurant ainsi une assez large dissémination pendant des siècles (surtout du XVIle siècle au début du XIXe siècle), malgré la concurrence d'autres granités régionaux de qualité (Ploujean, île de Batz et Ile Grande...)

\begin{abstract}
The discovery of very numerous quarries in the Callot granite, on the island-shore in Morlaix bay, the systematic examination of different buildings in the Morlaix country, and the archives data, point out, for the first time, the importance, in the past, of this pink stone, today forgotten. The spacial position of the buildings emphasizes the importance of the maritime transport, which has given to this granite a large enough dissemination during several centuries, in spite of the rivalry of other regional granites.
\end{abstract}




\section{L'extraction du granite rose de l'île Callot et son emploi dans le pays de Morlaix (Finistère)}

La reconnaissance de très nombreux anciens sites d'extraction du granice de Callot sur les estrans de l'île et les récifs voisins en baie de Morlaix, l'examen systématique des différents types de constructions (édifices religieux, châteaux et manoirs, habitat des cités et des campagnes...) dans toute la région morlaisienne, et les données d'archives (peu nombreuses), révèlent pour la première fois l'importance prise dans le passé par cette belle pierre rosée, à présent bien oubliée. La répartition spatiale des constructions souligne le rôle majeur joué par le transport maritime (baie de Morlaix prolongée par les rias du Dossen et de Penzé) de ce granite, lui assurant ainsi une assez large dissémination pendant des siècles (surtout du XVIIe siècle au début du XIXe siècle), malgré la concurrence d'autres granites régionaux de qualité (Ploujean, île de Batz et Ile Grande...)

The discovery of very numerous quarries in the Callot granite, on the island-shore in Morlaix bay, the systematic examination of different buildings in the Morlaix country, and the archives data, point out, for the first time, the importance, in the past, of this pink stone, today forgotten. The spacial position of the buildings emphasizes the importance of the maritime transport, which has given to this granite a large enough dissemination during several centuries, in spite of the rivalry of other regional granites.

* Directeur de recherche au C.N.R.S., Université de Bretagne occidentale, Brest. 
Louis ChAURIS

Dans le passé, les difficultés du transport par charrois des pierres de construction ont souvent conduit à l'ouverture de perrières proches des chantiers, qui - bientôt rebouchées - ont généralement disparu sans laisser de traces ${ }^{(1)}$. En l'absence de roches de qualité proximales, le cout des convois par les mauvais chemins augmentait considérablement le prix de revient ${ }^{(2)}$. $C^{\prime}$ est dire tout l'intérêt que pouvaient présenter alors les acheminements par voie d'eau $^{(3)}$. Du fait de sa configuration géographique, la Basse-Bretagne offrait, sous cet angle, des avantages exceptionnels. Par ailleurs, par suite de la nature géologique de son sous-sol, cette même région présente en bordure de mer différents granites de grande qualité architecturale. Enfín, sur le littoral, les roches sont, le plus souvent, débarrassées de leur manteau d'altérites ou de limons et alors directement exploitables sans longs et coateux travaux de déblaiement.

La conjonction de ces trois facteurs naturels favorables : présence littorale de roches de qualité ; extraction aisée par débitage immédiat des boules éparses sur les estrans ou des masses rocheuses en place dans les basses falaises et les îlots ; possibilité d'acheminement par voie d'eau, mer et abers, facilitant la pénétration à l'intérieur des terres, a suscité depuis longtemps l'extraction et le transport à distance des beaux granites côtiers bretons. Le cas du célèbre district de l'Ile Grande en Trégorrois a été étudié récemment ${ }^{(4)}$; le transport de son granite est attesté non seulement dans les ports bretons (à Tréguier dès la fin du Moyen Age ${ }^{(5)}$ ), mais aussi sur les côtes françaises de la Manche et de l'Atlantique et même à l'étranger (Belgique, Iles Britanniques). Le granite de l'Aber-Ildut, extrait depuis des siècles sur les rives de la ria et sur le littoral à proximité de son embouchure, a connu également une renommée nationale (socle de l'obélisque de Louqsor, place de la Concorde à Paris) et même internationale (quais de la Tamise à Londres) ${ }^{(6)}$. La pénétration aisée du Kersanton, depuis les diverticules du fond de la rade de Brest jusque dans le

(1) J.L. PORHEL. Les chantiers paroissiaux dans le Léon du XVIe au XVIIIe siècle. Mém. Maîtrise, Univ. Bretagne Occidentale, Brest, 1982, 157 p. (sous la direction de J. Tanguy).

(2) J.P. LEGUAY. «L'approvisionnement des chantiers bretons en matériaux de construction aux XIVe et XVe siecles». in «Pierre - Metal dans le bâtiment au Moyen Age». Paris, 1985, 372 p. (cf. p.27-79).

(3) Par la mer ou par les fleuves. Voir par exemple R. BEDON. in Les carrières et les carriers de la Gaule romaine. Edit. Picard, 1984, 248 p. : «La plupart des villes qui bordaient le Rhin a l'époque romaine ont utilisé le calcaire extrait à Norroy et acheminé par la voie fluviale».

(4) L. Chauris. «Carrières au bord de la mer. Ile Grande et îlots voisins (Côtes-du-Nord)». $115 e$ Congrès nat. Soc. savantes, Avignon, 1990. Colloque «Carrières et constructions», 1991, p. 305321. L. Chauris. «Le transport par mer des granites de l'lle Grande (Côtes-d'Armor)». Mém. Soc. Emul. Côtes-d'Armor, 1991, p. 75-90.

(5) M. Chaou. Une cité médiévale : Lantreguer au XVe siècle. Mém. de Maîtrise, Rennes, 1969, $200 \mathrm{p}$.

(6) L. Chauris. «Les carrières et les quais de chargement du granite rose de l'Aber-Ildut (Finistère)». Les Cahiers de l'Iroise, 150, 1991, p. 69-78. 
Léon par la rivière de Landerneau, est en partie à l'origine de la célébrité des enclos paroissiaux dits fort justement de la «Vallée de l'Elorn». Le microgranite de Logonna, extrait aussi en rade de Brest, offrait des possibilités d'acheminements comparables. Les pavés en microgranite de l'Ile Longue étaient transportés à Brest, jusqu'à Quimper et au-delà... Moins connus sont les exemples du granite de Trégunc extrait des bords de la ria de l'Aven(7), du beau granite gris clair de l'île de Batz, transporté déjà à Morlaix au moins dès le XVe siècle ${ }^{(8)}$, du granite de Cléder... ${ }^{(9)}$.

Dans ce mémoire, nous nous proposons de présenter succinctement le résultat de nos recherches sur les anciennes exploitations du beau granite rose rougeâtre de l'île Callot en baie de Morlaix (Finistère) et sur son utilisation pendant plusieurs siècles dans toute la région. Nos travaux révèleront, pour la première fois, l'extraordinaire ampleur prise par les extractions littorales dans cette petite île (moins de $2,5 \mathrm{~km}$ du nord au sud) et dans les îlots et récifs qui la frangent : au total, plus de 80 sites abandonnés ont été mis en évidence ! Nous nous attacherons à préciser la localisation des perrières ${ }^{(10)}$, les modalités de l'abattage du granite, le rôle joué par les rias de Morlaix et de Penzé pour la diffusion régionale du matériau, la variété étonnante des édifices ayant fait appel à ce granite.

Notre méthode d'investigation a privilégié les observations sur le terrain : relevé complet de tous les sites d'extraction ${ }^{(1)}$; examen aussi systématique que possible des constructions dans un large rayon autour de la baie de Morlaix, en vue de préciser les limites spatiales d'emploi de la roche. Le report de ces différentes données sur des cartes visualisera immédiatement les résultats obtenus et évitera, en même temps, d'allonger démesurément le texte. La comparaison des cartes entre elles fera ressortir les limites qu'il n'était guère possible de transgresser pour des raisons économiques et aussi par suite de la concurrence des autres granites régionaux. Dans plusieurs cas, les résultats acquis sur le terrain par l'étude pétrographique des monuments eux-mêmes seront complétés par des données d'archives - au demeurant, dans l'ensemble, ici relativement rares. Ainsi, des pans entiers d'une histoire régionale totalement méconnue seront peu à peu dévoilés, embrassant à la fois l'archéologie des perrières et l'architecture des anciennes constructions.

(7) Y.M. FouRnIS. «Les richesses du sous-sol finistérien», Le Consortium breton, I, vol. I, $\mathrm{n}^{\circ} 3$, 1927, p. 302-310.

(8) L. Chauris. «Quand l'île de Batz exploitait son beau granite gris». Le Courrier du Léon, $\mathrm{n}^{\circ}$ des 9,16 et 23 janvier. 1993.

(9) L. Chauris, «Naguère à Cléder, petite capitale du granite». Courrier du Léon des 29/05, 12 et $26 / 06 / 93$.

(10) «Perrière»: ancien nom de «carrière». «Rompeur», ancien terme pour «carrier».

(11) A l'échelle au $1 / 20.000^{e}$ de la belle carte de la baie de Morlaix éditée par le Service hydrographique de la Marine. 


\section{Louis CHAURIS}

\section{I - Les sites d'extraction L'île Callot ou les chantiers des rompeurs}

Avant d'aborder l'examen détaillé des anciennes exploitations, il importe d'évoquer rapidement les grandes lignes de la géologie du granite de Callot ${ }^{(12)}$. Cette roche représente le faciès le plus précoce du massif granitique dit de la baie de Morlaix - dont elle constitue la partie nord-occidentale ${ }^{(13)}$ - caractérisé par un gros grain, localement légèrement porphyroïde, la teinte rose rougeâtre du feldspath et le noir brillant du mica ; en quelques points (le Cerf, le Four...), la vive coloration du feldspath fait place à une nuance blanc bleuté. Aux affleurements, le granite de Callot apparaît fréquemment sous forme de boules qui confèrent aux rivages de l'île leur aspect pittoresque ${ }^{(14)}$. Dans les basses falaises littorales, l'espacement des diaclases isole de larges bancs massifs - jusqu'à 4 à 5 mètres de puissance - qui ont permis l'extraction de superbes monolithes. Dans les zones où les diaclases sont très rapprochées, les extractions sont inexistantes. La couleur agréable de la roche, son aptitude au façonnement et sa grande résistance à l'érosion constituaient autant de facteurs intrinsèques favorables à une exploitation intensive. Le comte de La Fruglaye, pionnier des Sciences de la Terre dans le nord-ouest de la Bretagne, semble avoir été le premier à souligner les qualités ornementales du granite de Callot qui, au poli, acquiert une «teinte charmante et un vif éclat» ${ }^{(15)}$.

Les anciens chantiers d'extraction peuvent être regroupés en trois districts d'importance très inégale. Le principal - et de loin - est situé à l'île Callot et les nombreux récifs qui ourlent l'île à proximité (fig. 1): nos investigations ont permis ici la reconnaissance de 65 perrières différentes, avec des zones de forte densité où les chantiers sont pratiquement jointifs (entre An Ode Velen et Beg Kraou Denved sur la côte nord-ouest ; entre Vougeo et Pennenez sur la côte nord-est, aux environs de Porz Moualc'h sur la côte sud-est). En fait, les zones dépourvues d'extrac-

(12) L. Chauris. «Succession des intrusions dans le complexe granitique tardi-hercynien de la baie de Morlaix (Massif Armoricain)». C.R. Acad. Sci. Paris, 286, 1978, p. 253-256. Du même auteur (1986). In Notice explicative de la feuille géologique Plestin-les-Grèves à 1/50.000. Edit. BRGM, «Orléans : Complexe granitique de la baie de Morlaix», p. 33-36.

(13) Les autres faciès du massif granitique de la baie de Morlaix sont représentés par le granite à grain moyen à fin, de teinte ocre, dit de Carantec ; le granite fin rougeâtre de Plougasnou ; le granite blanc à grain fin de Sterec; et le granite porphyroïde blanc de Saint-Samson.

(14) Cette morphologie en boules n'est pas sans rappeler l'aspect présenté aussi par les granites de l'Aber-Ildut et de Ploumanac'h. Tous ces massifs granitiques appartiennent à la même ceinture, bien mise en évidence par $\mathrm{Ch}$. Barrois et connue sous le nom de «traînée moniliforme [du latin«monilis» = collier] des granites rouges» de Bretagne septentrionale, tous caractérisés par leur âge récent (environ 300 millions d'années).

(15) Onfroy-Kermoalquin, Foi bretonne du 8/01/1850. Sur l'œuvre géologique du comte de La Fruglaye, voir L. ChauRIS. «Un pionnier des Sciences de la Terre en Bretagne Occidentale : le comte de La Fruglaye (1766-1849)». Bull. Soc. archéol. Finistère (sous presse). 


\section{L'extraction du granite rose de l'île Callot...}

tion granitique correspondent essentiellement aux affleurements de panneaux gneissiques (entre Run Lann et les Platines) ${ }^{(16)}$; à certaines grèves sans socle rocheux (Aber ar Biliou) ou encore aux secteurs faillés (entre Ar Run et An Ospital). Certains récifs, situés parfois à plusieurs centaines de mètres de l'île et uniquement accessibles à basse mer, ont été aussi exploités plus ou moins intensément (Men Mat, le Four, Enez Wenn, Run Lann, les Platines...). Le second district - que nous appellerons de l'Ile Verte, du nom du principal îlot - s'étend au nord-est de Callot et est uniquement accessible par bateau (fig.1). Il regroupe les récifs du Cerf, de la Pierre Jaune, les deux îlots de l'Ile Verte et également, selon toute probabilité, Ar Vezou, où les traces d'extraction sont moins nettes. Ces deux premiers districts appartiennent à la commune de Carantec. Un troisième et dernier district apparaît au sud-ouest de l'île Callot, par delà le chenal de la Penzé, aux environs de la presqu'île de Trégondern, en SaintPol-de Léon, principalement autour de l'île Blanche, entre Men ar turh et Toull Houarn (fig. 2). À part la petite occurrence de Trégondern, tous les anciens chantiers sont ici «insulaires». Certains d'entre eux, isolés au milieu des vasières, sont totalement recouverts à marée haute. Seules émergent un peu plus largement l'Ile Blanche et Toull Houarn. À notre connaissance, les enquêtes toponymiques ${ }^{(1)}$ n'ont pas identifié le terme «mengleuz» (carrière) dans les trois districts précités. Toutefois, la dénomination «Men Mat» (Bonne pierre) pour une zone rocheuse située à l'ouest de Callot se réfere peut-être à l'excellente qualité du matériau extrait ici.

Les modalités présentées par les extractions sont fonction de la position par rapport au niveau de la mer, de l'étendue des chantiers - depuis la simple grosse boule jusqu'aux masses rocheuses en place - et enfin des méthodes d'abattage, elles-mêmes sous la dépendance, en première approximation, des périodes d'exploitation. De très nombreux chantiers étaient localisés sur l'estran lui-même - c'est-àdire dans la zone de balancement des marées - parfois même jusqu'à la limite infé-

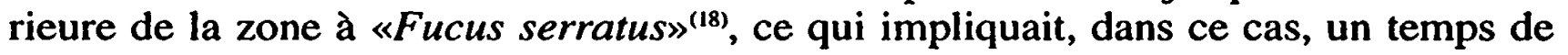
travail singulièrement limité par le rythme bi-journalier de la marée. D'autres perrières étaient situées dans les basses falaises elles-mêmes et ici, seules les parties supérieures échappaient à l'action de la mer. Enfin, beaucoup plus rarement, les chantiers étaient ouverts au-dessus du niveau des plus hautes mers (localement à l'Ile Verte et à Roc'h Losket, près du fort de Callot, à la pointe au nord de Vougeo...). La rareté relative de tels cas s'explique aisément : les avantages des carrières littorales au sens strict disparaissaient ici (altération possible, transport plus difficile...). En fait, dans plusieurs cas, toutes les transitions existent entre les chantiers ouverts sur l'estran et les extractions «à terre».

(16) Les gneiss ont été également recherchés jadis pour l'obtention de moellons allongés, très utilisés dans les vieilles constructions à Carantec.

(17) A. LE BERRE. «Toponymie de la baie de Morlaix». Extr. des Annales hydrographiques (1969), p. 325-399. Le toponyme "Karreg ar Vengleuz» (= Roche de la Carriere) dans le récif des Duon se rapporte à une roche schisteuse.

(18) Sur l'estran, les algues (goémons) se répartissent en zones verticales, en fonction du niveau atteint par la mer. 


\section{Louis Chauris}

Le débitage des boules disséminées sur l'estran a certainement été très important au vu des vestiges encore conservés : boules ayant fait seulement l'objet d'une tentative de fente, énormes boules partiellement exploitées... L'extraction des masses rocheuses en place dans les basses falaises était facilitée par la disposition des diaclases, systèmes de fissures débitant la masse granitique selon trois directions. De nombreux chantiers se sont ainsi arrêtés sur une diaclase peu inclinée qui constituait la «sole» ou plancher de la perrière. Le démantèlement systématique des masses rocheuses a conduit peu à peu à la formation d'un platier artificiel, parsemé de mares, parfois à morphologie «triangulaire», marquant l'emplacement des blocs extraits. Quelquefois, seulcs les têtes de roches ont été écrêtées. Au nordouest de l'Ile Blanche en Saint-Pol, l'extraction se marque par une sorte de tranchée dans la masse granitique.

L'extraction du granite de Callot pour l'obtention des pierres de taille s'est effectuée au moyen de deux procédés dont les traces sont encore bien visibles sur le terrain.

La méthode des coins est très ancienne. Le débitage des masses granitiques était obtenu par une succession rectiligne de «trous» alignés, emplacés à intervalles assez réguliers et destinés à recevoir des coins. Les zones où l'extraction en cours a été abandonnée permettent de reconnaître diverses variantes morphologiques de tels «trous» (ovoïdes, rectangulaires, très allongés...). À résistance égale de la roche et pour une morphologie originelle déterminée, il apparaît une relation entre la dimension des trous et leur âge. Les trous les plus anciens offrent, en première approximation, les plus fortes dimensions ; le processus d'agrandissement est dû uniquement à l'érosion naturelle (lente corrosion par l'eau de mer et, aussi, le sel). Dans quelques cas, les cavités tendent même à devenir jointives, au moins dans leur partie supérieure.

À titre d'information, on a reporté dans le tableau I quelques données chiffrées (successivement longueur (L), largeur (1) et espacement (e) des trous). Dans les exemples de ce tableau, la longueur des trous ne présente, dans chaque cas, que de faibles variations. Dans quelques rares cas, elle est, au contraire, très variable, comme l'indique l'exemple suivant ( $\mathrm{L}, 1$ et e) : $20-3,5-4 / 7-2,5-$ $5,5 / 7-2,5-8,5 / 25,5-2,5-7 / 7-2,5-7 / 7-2,5$. Parfois, certaines alvéoles sont exceptionnellement longues (jusqu'à $40 \mathrm{~cm}$, et même près de $50 \mathrm{~cm}$ à Enez Wenn). Au pied de la Tourelle Mazarin, on a observé une encoche en $V$ formant une sorte de rainurage de plus d'un mètre de long. De telles modalités de fente restent tout à fait sporadiques à Callot. Selon les plans de débitage du granite, les trous sont disposés verticalement ou, plus rarement, horizontalement. Dans quelques cas, les trous ont été creusés sur le même bloc selon deux directions perpendiculaires. Dans le cas d'extraction interrompue, il ne reste plus que des entailles, qui avec l'avancement de l'érosion, confèrent à la partie supérieure de la roche fendue, un aspect quelque peu dentelé. Les fentes «ratées» se traduisent par des cassures curvilignes. 


\section{L'extraction du granite rose de l'île Callot...}

La méthode des trous de perforation offre des modalités totalement différentes. À l'inverse des trous pour coin, toujours rapprochés et peu profonds, alignés au nombre d'une douzaine ou même plus, les trous de perforation, effectués par une longue barre, parfois dénommée dans le passé «chante-perce», sont généralement uniques, toujours circulaires et d'une profondeur de l'ordre d'un mètre. Une seule foration judicieusement implantée pouvait conduire à la fente verticale - ou plus rarement horizontale - du bloc granitique, de manière rectiligne ou, plus souvent, en dièdre très ouvert. L'examen de nombreux sites semble bien établir que la méthode d'abattage au moyen des trous de perforation (plus récente) entraînait plus de perte que la méthode des coins (plus ancienne).

L'étude des sites d'extraction indique que la poursuite des exploitations, parfois pendant plusieurs siècles, a entraîné de singulières modifications géomorphologiques dans le paysage primitif : à l'évidence, le littoral a été ici remodelé par l'action de l'Homme ; d'innombrables boules ont disparu sans laisser d'autres traces que parfois quelques débris de taille. Le démantèlement de l'estran rocheux et des falaises a eu une double influence : la formation d'un platier a tendu à l'aplanissement de l'estran, tandis que l'extraction dans les falaises a, au contraire, accentué leur pente naturelle. Parfois, comme à Roc'h Losket, de nombreux fragments abattus, anguleux, non utilisés, ont été laissés sur place ; en ce point, l'exploitation était par ailleurs gênée par de nombreuses enclaves de gneiss. Mais, peu à peu, surtout dans la zone de balancement des marées, la nature reprend ses droits : les plans de séparation des bancs granitiques tendent à revêtir un poli naturel, le platier de néo-formation se couvre de balanes et de patelles, les mares artificielles sont colonisées par les algues, les trous pour coin servent d'abris à une petite faune... $\AA$ terre, les débris de taille forment un sol rocailleux artificiel ; sur l'estran, ces mêmes fragments, qui se distinguent immédiatement des galets naturels par leur fraîcheur, tendent peu à peu à s'émousser. Localement, des blocs de lest abandonnés, de nature pétrographique totalement différente («schistes zébrés» de Morlaix) prouvent que des gabares venaient sur les estrans charger le granite extrait.

\section{II - L'emploi du granite de Callot au long des siècles}

En vue de déterminer l'utilisation du granite de Callot dans le passé, un inventaire systématique des différents types de construction dans toute la région de Morlaix au sens large a été entrepris sur le terrain : édifices religieux (églises, chapelles, bâtiments conventuels, calvaires), châteaux et manoirs, infrastructures portuaires, routières et ferroviaires, édifices divers, habitat. Les résultats de cette longue enquête sont reportés sur les cartes 3 à 5 . Trois remarques préliminaires doivent être faites dès l'abord. Le plus souvent, en l'absence de sources écrites, il ne sera pas possible de décider si le granite «type Callot» provient effectivement de cette île ou des îlots voisins (district de l'Ile Verte ou district de l'Ile Blanche) ; 


\section{Louis Chauris}

malgré cette incertitude, la proximité des trois districts ne peut que confirmer les conclusions relatives à la dissémination spatiale des constructions ; au demeurant, l'ampleur et le nombre des sites d'extraction à Callot privilégient à l'évidence ce dernier district. Par ailleurs, du fait de l'utilisation ancienne du granite étudié, il est évident qu'un certain nombre d'édifices qui l'avaient employé dans le passé ont disparu sans laisser de trace (au mieux la pierre a pu être remployée) ; les cartes qui présentent l'état des choses en 1992 ne figurent par suite que des minima. Enfin, malgré la densité des itinéraires de reconnaissance, plusieurs constructions ont échappé à notre enquête ; cependant, les résultats obtenus obéissent à une logique telle qu'ils doivent refléter la réalité d'assez près ; cette remarque concerne en particulier la détermination des limites spatio-temporelles d'utilisation du granite de Callot qui demeure l'un des buts majeurs de nos recherches.

\section{Édifices religieux}

Ce chapitre concerne les églises, les chapelles, les bâtiments conventuels et les calvaires (fig. 3). L'ampleur du sujet nous a contraint, sauf exception, à résumer les données obtenues lors de nos enquêtes.

Le célèbre Kreisker, à Saint-Pol-de-Léon, présente deux campagnes principales de construction (dernier quart du XIVe siècle et second quart du XVe siècle), suivies à plusieurs reprises de travaux de restauration, en particulier au XVIe siecle (1576), au $\mathrm{XVI}^{\mathrm{e}}$ siècle (1639) et au début du XIXe siècle $^{(19)(20)}$. Son édification a surtout fait appel à différents granites extraits aux environs de la ville (leucogranite à tourmaline, leucogranite à muscovite...) ; le granite type Callot a été aussi largement employé, en particulier dans l'élévation sud, où il se distingue facilement des autres granites par sa teinte plus sombre ; on le retrouve aussi au chevet et dans la plate-forme au sommet de la tour... À moins qu'il ne s'agisse de travaux de restauration, au demeurant fort improbables dans le cas de l'élévation méridionale, il semble bien que nous ayons ici la première utilisation de ce granite conservée jusqu'à nos jours, dans un édifice religieux.

L'église Saint-Melaine à Morlaix remonte à la fin du XVe siècle et au XVIe siècle $^{(21)}$. Dans l'élévation méridionale, à l'est du porche, on note un assez large

(19) Sans compter les importants travaux commencés sur la flèche en 1992.

(20) Les données chiffrées relatives aux édifices religieux cités dans cet article sont, en règle générale, extraites du remarquable ouvrage de R. Couffon et A. LE BARS : Diocèse de Quimper et de Léon. Nouveau répertoire des églises et chapelles. Quimper, 1988, 552 p. Pour le Kreisker et la cathédrale de Saint-Pol, voir Également L. Th. LÉcureux : Saint-Pol-de-Léon. La Cathédrale Le Kreisker, Paris 1925, 96 p., et Y.P. CASTEL : Saint-Pol-de-Léon, Rennes, 1979. Sur la nature lithologique des édifices religieux du nord-est finistérien, consulter $\mathbf{L}$. CHAuris : «Geologie et histoire de l'architecture : la provenance des pierres utilisées dans la construction des édifices religieux de la région morlaisienne». Bull. Soc. archéol. Finistère, 1993, t. CXXII, p. 225-273.

(21) Y.P. CASTEL. «Le demi-millénaire de l'église Saint-Melaine de Morlaix (1489-1989)». Les cahiers de l'Iroise, 35e année, 1988, p. 77-87. 
L'extraction du granite rose de l'île Callot...

emploi du granite rose de Callot en association avec le granite gris de l'île de Batz, prédominant ; mais il s'agit ici d'une restauration datant probablement du début du XVII' siècle. L'ajout assez malencontreux, édifié en 1783 à l'ouest du même porche, est également en granite de Callot.

En fait, dans les édifices religieux antérieurs au XVIIe siècle, le granite de Callot a été rarement reconnu. À Saint-Jean-du-Doigt (XVe siècle et début du XVI ${ }^{\mathrm{e}}$ siècle), cette roche reste très sporadique (dans un contrefort de la tour, sous une fenêtre méridionale). Dans la vieille église de Taulé (XVIe siècle) dont seul subsiste le clocher, le granite de Callot forme divers éléments. Dans la chapelle SaintVissias en Guiclan, paraissant remonter au XVIe siècle, mais ultérieurement très remaniée, le granite de Callot demeure rarissime.

Au XVIre siècle, l'emploi du granite de Callot devient plus important. À Locquénolé, l'édifice qui remonte en majeure partie à cette époque, a utilisé cette roche en particulier dans le porche ; il est significatif de noter son absence totale dans les parties romanes conservées - où apparaissent des grès tuffacés et des microgranites locaux. À Henvic, l'ancienne église en ruines, rapportée au XVIIe siècle (avec sans doute des parties plus anciennes ?) montre un large emploi du granite de Callot. L'église de Guiclan, reconstruite en 1878-1880, a conservé plusieurs éléments de l'ancien édifice (porche de 1615, chevet de 1688). L'utilisation ancienne du granite de Callot, observé en plusieurs points (intérieur de l'église sous la tour...) en pierres de grand appareil, est confirmée par les données d'archives ${ }^{(22)}$. Dans l'église de Ploué$z o c^{\prime} h$ (XVII $^{\mathrm{e}}$ siècle), le granite de Callot a été également noté en plusieurs points. Comme on pouvait a priori s'y attendre, la chapelle N.D. de Callot, reconstruite à plusieurs reprises et en particulier au XVII ${ }^{\mathrm{e}}$ siècle (la tour porte la date de 1672) a très largement fait appel au granite local ; toutefois, la base de la façade ouest et le porche occidental ont utilisé le granite clair de l'Ile Grande ${ }^{(23)}$, en net contraste avec le granite de Callot plus sombre.

Au XVIII e siècle, le granite de Callot est toujours utilisé, au moins pour partie, dans les constructions religieuses de la région morlaisienne. C'est à cette époque que se rapportent l'église de Saint-Sève bénie en 1753 ; la façade occidentale de la chapelle Saint-Pierre dans le cimetière de Saint-Pol (1772) ; la chapelle du château de Bagatelle (1772) ; la façade de l'église de Penzé (1789) ; la chapelle Sainte-Anne du Fransic en Carantec (1789) et, comme on l'a vu, l'ajout méridional de Saint-Melaine (1783). L'église Saint-Martin de Morlaix (pose de la première pierre en 1775, bénédiction en 1788) mérite une mention spéciale par sa large utilisation du granite de Callot (pour partie) : piliers, entrée intérieure du porche sous le clocher, portes, élévations nord et sud..., en association avec le granite de Pont-Pol, extrait un peu au sud de Morlaix et d'autres granites.

(22) «Comptes de fabrique 1685-1688», relevés par J.L. Porhel (op. cit. note 1) dans les Archives départ. Finistère $76 \mathrm{G} \mathrm{4,13}$ et 21 .

(23) G. LuCE-LozAC'H. Carantec. Une cité côtière du Léon, 1989, 208 p. Voir p. 117. 


\section{Louis Chauris}

Au XIXe siècle, l'emploi du granite de Callot dans les édifices religieux se ralentit. Notons au passage son utilisation sporadique dans la chapelle du cimetière Saint-Charles à Morlaix (1828), dans la chapelle du Frout à Carantec (1860), son utilisation plus importante dans le chevet de la chapelle du collège du Kreisker, en association avec le granite de l'île de Batz - Roscoff. Deux exceptions remarquables doivent toutefois être relevées. La première concerne la reconstruction de l'église Saint-Mathieu à Morlaix (seule la tour, édifiée dans la seconde partie du XVIe siècle avec des granites de Plourin, de l'Ile Grande, de l'Ile de Batz, a été conservée) entre 1823 et 1827 , qui a largement employé le granite de Callot dans le portail d'entrée à gauche de la tour, les piliers, les portes (l'unc d'elles avec la date de 1826)... La seconde exception se rapporte à l'église paroissiale de Carantec. La nature des pierres de l'ancienne église, édifiée en 1657 et démolie en 1866, nous reste inconnue. Le nouvel édifice, achevé en 1867 (sauf le clocher qui date de 1887) a largement employé le granite de Callot en particulier pour les contreforts, la base des piliers, la sacristie..., auquel s'adjoignent les granites de Guerlesquin, de Cléder et de l'Ile Grande, ces deux derniers transportés par mer. Les charrois, depuis les grèves jusqu'au bourg étaient assurés par les paroissiens ; les équipes de service étaient désignées à la messe du dimanche. «M. le Maire, de concert avec M. le Recteur [avait] fait venir de Bordeaux une barrique d'eau-de-vie pour être distribuée pendant la construction de l'église aux prestataires qui feront gratuitement les charrois des matériaux» ${ }^{(24)(25)}$.

Le granite de Callot a été également observé dans plusieurs bâtiments conventuels : aux Jacobins à Morlaix (dans l'élévation sud de l'ancienne caserne) ; aux Ursulines de la même ville (XVII' siècle) ${ }^{(26)}$. Le couvent de Cuburien est particulièrement intéressant pour notre propos. Dans la partie orientale de la superbe façade méridionale du bâtiment, portant la date de 1560, le granite de Callot n'a pas été noté ; par contre, dans la partie de la même façade située plus à l'ouest et portant la date de 1677, cette roche a été largement employée (en association avec le granite de Ploujean). Dans ce cas précis, le granite de Callot n'est pas utilisé au XVIe siècle, mais uniquement dans la seconde partie du XVII ${ }^{e}$ siècle.

Quelques calvaires ont employé le granite de Callot, mais, semble-t-il, dans l'état actuel des recherches, uniquement pour la partie inférieure ; par contre, le fût et la croix sont de nature pétrographique différente. Ainsi, près de l'église de Carantec, sur un socle monolithe en granite de Callot se dresse un fat en diorite (de provenance imprécisée) surmonté d'une croix en kersanton (1839) ; toujours à

(24) Archives municipales de Carantec.

(25) L'église de Plouénan (1884-1887) remplace un édifice de 1770 . Il ne nous a pas encore été possible de savoir si les moellons en granite de Callot, assez fréquents dans l'élévation sud (en association avec le granite de Sainte-Catherine) proviennent d'un remploi de la construction primitive ou représentent une première utilisation. C'est ici, à notre connaissance, le seul édifice religieux où le granite de Callot a été utilisé en moellon et non en pierre de taille.

(26) A. Daniellou. À travers 340 ans d'histoire. Les Ursulines de Morlaix. 1980, Morlaix, 226 p. 


\section{L'extraction du granite rose de l'île Callot...}

Carantec, la base (trois degrés et socle carré) de la Croix du Salut (XVIIe siècle) est en granite de Callot, surmontée d'une croix récente en granite de Huelgoat. D'autres exemples ont été notés à Taulé, près de l'église (fût en granite de Cléder et croix en kersanton); toujours à Taulé, à la cote 90 près du bourg (kersanton); à Morlaix, près de l'église Saint-Mathieu, la croix de Mission de 1949 a remployé une base en granite de Callot...

Le granite de Callot a dû aussi être recherché dans le passé pour la confection des pierres tombales. Toutefois, par suite du déplacement des cimetières et de la destruction - fort regrettable - de la plupart des tombes anciennes, cc type de monument est aujourd'hui très rare. Une dalle tumulaire façonnée en ce granite repose au milieu de l'herbe dans l'ancien cimetière d'Henvic à proximité de la vieille église.

\section{Châteaux et manoirs - ouvrages défensifs}

De nombreux manoirs et quelques châteaux ont fait appel au granite de Callot, quelquefois largement, souvent d'une manière sporadique (fig.4). Dans plusieurs cas, les destructions et les remaniements successifs ont modifié les édifices primitifs, dont la nature pétrographique reste alors délicate à préciser.

Le château du Penhoat en Saint-Thégonnec - «fantôme féodal drapé dans son linceul de lierre»(27) — qui remontait au XIII' ${ }^{(2)}$ siècle, a été démoli pendant la Ligue ; les ruines montrent des murailles très épaisses en micaschistes de provenance locale ; dans une ouverture a été noté un peu de granite de Callot, sans que l'on puisse toutefois assurer avec certitude que son utilisation remonte à la construction primitive. Jouxtant le manoir de Trogriffon, enfoui dans son cadre de verdure au fond d'un diverticule de la Penzé, une vieille tourelle (XIVe siècle ?) en moellons de micaschistes d'origine proximale, montre une porte avec plusieurs éléments en granite de Callot ; mais la même incertitude qu'au Penhoat règne ici sur l'époque précise de leur pose. La jolie maison prébendale de Saint-Pol-de-Léon fort bien conservée - date de la première partie du XVIe siècle ; outre le granite de l'Ile de Batz, prédominant, sa construction a aussi utilisé localement le granite de Callot. Le manoir en mauvais état de Lannoverte en Plouézoc'h, également du XVIe siècle, a fait appel au granite de Ploujean et le granite de Callot reste ici subordonné. Le manoir de Kerangoaguet en Carantec (XVIIe siècle) présente plusieurs éléments en granite de Callot, tranchant sur les moellons de grès-quartzites grisâtres de provenance locale ; le magnifique portail d'entrée (de la même époque) en granite de Callot a été démonté et reconstruit devant l'église paroissiale de Carantec vers 1935. L'ancienne maison manale de Vorlenn en Taulé conserve une vieille porte en granite de Callot portant la date de 1603. Dans le château de Kerangomar en Taulé (XVIIe

(27) L. Le Guennec. Le Finistère monumental. T. 1. 1979, Morlaix et sa région, 416 p. 


\section{Louis ChaURIS}

siècle), le granite de Callot est associé au granite de Ploujean. Dans le château de Coatilès (XVII' siècle), également en Taulé, le granite de l'Ile Grande a été très largement employé et le granite de Callot reste rare.

Dans de nombreux cas, il est difficile de préciser l'âge des bâtiments (XVIe ou XVII ${ }^{e}$ siècle ?), peut-être même parfois plus anciens, voire plus récents ? Dans ce groupe se placent, parmi d'autres : à Carantec : Kerhallic (avec une superbe porte cintrée bien ouvragée), Cosquer Ven (pierres de la façade méridionale en grand appareil, beau linteau à double accolade...), Plouzornou (pierres d'angle, porte cintrée), le Frout... ; à Henvic : Lezireur (restes, remanićs, de l'ancienne demeure, incendiée en 1594), Quistillic (portes), Lingoz... ; à Taulé : Kergadoret (belles pierres de taille), Kerollac'h (rare), Kergus, Kerassel... ; à Ploujean : Mousterou, le Launay (dans des ouvertures)... ; à Plouézoc' $h$ : Brignonic (avec ses grès tuffacés), Keryvoalen (avec son escalier extérieur), Baradozic (certainement antérieur à 1640) ; à Saint-Sève : Le Quinquis (rare) ; à Saint-Martin-des Champs : Bréventec ; à Guiclan : Kermorvan (surtout remarquable par ses grands éléments schisteux).

Au XVIII siècle, le granite de Callot a été utilisé, parfois assez largement, comme dans le manoir du Fransic en Carantec et dans le château de Bagatelle en Saint-Martin-des-Champs, ou, au contraire, sporadiquement comme dans les châteaux de Keranroux en Ploujean et de Kervolongar en Garlan. Il est très abondant dans l'édifice dit de «Cornic» au Bas de la Rivière en Ploujean (extrême fin du XVIII siècle ?), où il est associé au granite de l'Ile Grande. Selon L. Le Guennec $^{(27)}$, le style du manoir de Trogriffon en Henvic (mise à part la vieille tourelle que nous avons déjà évoquée) indique le XVIIIe siècle ; l'emploi du granite de Callot est également ici assez fréquent. L'aile sud des bâtiments de l'ancien palais épiscopal de Saint-Pol-de-Léon (actuellement occupé par l'Hôtel de Ville), édifiée au milieu du XVIII ${ }^{e}$ siècle (1750), montre un large appel au granite de Callot en pierres de grand appareil.

Bien que de destination différente, les ouvrages défensifs sont regroupés dans le chapitre relatif aux châteaux. Des remparts qui protégeaient Morlaix, il ne demeure plus à présent que quelques vestiges, où le granite de Ploujean occupe une large part ; toutefois, à la partie supérieure de ces murailles, le granite de Callot a été également observé, sans que l'on puisse assurer avec une certitude absolue si son emploi remonte ici au XVe siècle. Sur le monticule qui domine l'extrémité septentrionale de l'Ile Callot, les restes d'un vieux fort en pierres de grand appareil, en granite de l'île, apparaissent encore au milieu des landes ; certains

éléments de la fortification ont été probablement remployés ailleurs... Le granite de Callot a été très recherché - et c'est sans doute ici que se place son utilisation majeure - lors de la construction du célèbre château du Taureau, édifié par les Morlaisiens, aux XVII' et XVII' ${ }^{e}$ siècles, à l'entrée de la rade de Morlaix, pour protéger leur ville des incursions anglaises ; le granite de Callot, en grand appareil, confère à la forteresse une teinte très caractéristique ; le granite gris de l'île de Batz est resté très subordonné. 
L'extraction du granite rose de l'île Callot...

\section{Constructions diverses}

Sous cette rubrique ont été regroupés les infrastructures portuaires au sens large (quais, jetées, cales), les infrastructures routières et ferroviaires, divers édifices urbains, l'habitat des cités et des campagnes... ayant fait appel au granite de Callot (fig. 5). À Saint-Pol-de-Léon et surtout à Morlaix, où les maisons en granite de Callot sont nombreuses, l'emploi de symboles individuels était évidemment impossible, d'où le regroupement à l'intérieur d'un cercle. Enfin quelques utilisations très particulières (puits, auges...) ont été également signalées. La description détaillée de ces constructions variées deviendrait vite fastidieuse ; aussi, nous bornerons nous ici à présenter seulement quelques exemples de chaque type particulièrement intéressants.

\section{Infrastructures portuaires}

\section{Port de Morlaix}

Les archives municipales de cette ville conservent un dossier en date du 20 mars 1751, relatif au «Devis des ouvrages des quay et calles du costé de Tréguier» pour l'aménagement du port ${ }^{(28)}$. Il est stipulé que «les pierres de taille qui seront employées à la construction de ces ouvrages seront tirées des meilleures carrières de l'Isle Callot et des Isles voisines, choisissant celles qui ne sont point roussâtres ; elles seront proprement taillées sur leur parement... L'entrepreneur ne sera tenu de commencer à y travailler qu'à l'entrée de la belle saison de 1752, mais au moyen des avances qui lui seront faites... il fera tirer de la pierre de taille à force dans les carrières de Callot, profitera de tout le temps que l'on pourra naviguer pendant le cours de la présente année, pour cn faire transporter le long du quai de Tréguier, le plus qu'il sera possible...». Concernant toujours les «quay et calles de Morlaix», les archives municipales en date du 4 avril 1762 citent encore la «toise quarree de parement de pierre de taille de l'Isle de Calot». Le 20 de ce même mois, une fois de plus pour la continuation du quai de Tréguier, on demande l'emploi de «tous les morceaux de pierre de l'Isle Calot, tant taillée que non taillée qui se trouvent sur le quai et aux environs»(29).

(28) Archives municipales de Morlaix, série DD. Les modalités de la construction et les prix sont fixés d'une manière très précise dans le devis : «La toise quarrée de parement de pierre de taille de l'Isle de Calot, mise en place avec mortier fin, composé de moitié chaux... éteinte, de moitié sable de mer bien dessalé, passé au crible, sans diminution du cube de la dite pierre, à 29 livres 15 sols».

(29) D'autres données complémentaires sont disponibles dans les archives départementales d'Ille-et-Vilaine C 664 à C 668 et C 1180 . Voir aussi le Mémoire de Maîtrise de F. YSNEL, 1988 : l'aménagement du port et de la baie de Morlaix au XVIIIe siècle. Univ. de Haute-Bretagne, $187 \mathrm{p}$. 


\section{Louis Chauris}

\section{Port de Roscoff}

Dans le vieux port, la jetée centrale, à l'approche du musoir et au musoir luimême, montre un large emploi du granite de Callot en pierre de taille de grande dimension, associé aux granites de Roscoff et de Cléder.

\section{Infrastructures routières et ferroviaires}

À Morlaix, les escaliers montant à l'église Saint-Melaine montrent l'association du granite de Callot au granite de l'île de Batz... (le granite du Huelgoat se rapporte à une rénovation récente). Dans le parapet des escaliers accédant aux Ursulines de la même ville, le granite de Callot a été largement utilisé. La même roche, usée par le passage, se retrouve aussi dans des escaliers extérieurs à Penzé... À plusieurs reprises, les archives attestent également l'utilisation du granite de Callot pour des travaux de voirie au sens large. Un nommé François Hervé reçoit en 1722, 56 livres 14 sols pour la pierre de Callot en vue de «raccomoden le chemin par où allait venir le duc de Fitz-James lors de la tenue des États de Bretagne ${ }^{(30)}$. Les archives municipales de Morlaix ${ }^{(31)}$ en date du 12 janvier 1778 conservent le devis d'un parapet-barrière et autres ouvrages à exécuter en haut de la rue Courte et en haut des escaliers de la rue Fitz-James : «tirage à Calot des 18 pierres et leur port à Morlaix à 7 livres $10 \mathrm{~s}=135$ livres».

Le célèbre viaduc de Morlaix, construit entre 1861 et 1863 a nécessité $65.830 \mathrm{~m} 3$ de maçonnerie ${ }^{(32)}$. Une part importante des pierres de taille et des moellons piqués a été extraite des carrières littorales des environs de l'Ile Grande et transportée par mer jusqu'au chantier ${ }^{(33)}$. Une certaine quantité de pierres de taille provient également de l'île Callot ; ces pierres, en superbes éléments, ayant parfaitement résisté aux intempéries, sont encore bien reconnaissables dans l'ouvrage d'art. Les extractions du granite de Callot pour le viaduc paraissent bien représenter l'ultime participation importante de ce beau granite aux constructions morlaisiennes ${ }^{(34)}$.

\section{Édifices urbains}

La construction de l'ancien Hôtel de Ville de Morlaix, au XVII ${ }^{e}$ siècle, a fait appel au granite de Callot ${ }^{(35)}$. La construction traînait en longueur par suite du formalisme de l'Administration. «Fallait-il avoir une batelée de pierre..., les juges assistés de leur greffier et accompagnés de notaires, se transportaient sur les lieux...

(30) Arch. nat. H 523.

(31) Arch. munic. Morlaix, série DD.

(32) V. FEnOux. «Note sur les travaux de construction du grand viaduc de Morlaix». Ann. des Ponts et Chaussées, $\mathrm{n}^{\circ} 150,1867$, p. 207-236.

(33) Op. cit. note (4).

(34) Une proportion importante des moellons de blocage à l'interieur des piles du viaduc provient de différentes zones rocheuses de la baie de Morlaix, mais pas nécessairement de lî̀le Callot (granite de Carantec sensu stricto, granite de l'Ile Stérec). (Arch. municipales de Carantec en date du 15 juin 1862). Voir aussi L. Chauris,. «Coupeurs de goémon contre tailleurs de pierre. Cas de lutte pour la possession de l'estran en Bretagne au XIXe siecle». Ann. de Bretagne 100, 1, 1993, p. 121-127.

(35) G. LE JEAN. Histoire politique et municipale de la ville et de la communauté de Morlaix. Morlaix, 1846, 260 p. Le granite «se tirait de Callot». 
L'extraction du granite rose de l'île Callot...

à Callot..., on dressait des procès-verbaux bien détaillés... de la bonne qualité de la pierre... des procès-verbaux de reconnaissance constataient l'état des matériaux vendus sur les lieux»(36). Une partie des pierres de cet édifice, en provenance de Callot, sera réutilisée lors de la construction de la nouvelle mairie dans la première partie du XIXe siècle. «M. le Maire, toujours dans des vues d'économie bien ou mal entendues, voulut que les matériaux des anciens édifices servissent dans le nouveau de préférence à tous les autres... Chacun dans le public s'étonnait de voir des pierres de différentes couleurs dans un édifice qui devait faire honneur à la ville» ${ }^{(37)}$. Encore aujourd'hui, l'observation des murs de la mairie ne fait quc confirmer ce polylithisme assez curieux (en particulier association du granite de Callot avec le granite de Pont-Pol, localité située un peu au sud de Morlaix).

Le bâtiment de l'hôpital de Morlaix, édifié au-dessus du Queffleuth entre 1733 et 1737 a fait un grand emploi du granite de l'Ile Callot. Le premier ensemble de bâtiments de la manufacture des tabacs de la même ville, sur le quai de Léon, date de la même période (1736-1740). Pendant la construction de ce bel ensemble architectural de l'époque Louis XV, le granite de Callot était amené par voie d'eau devant le chantier et livré à «une armée de tailleurs de pierre» (38). Les blocs de granite déchargés dans le port étaient, en effet, semble-t-il, bruts. C'est ce que laisse aussi entendre ultérieurement Bigot de Morogues ${ }^{(39)}:$ «J'ai vu dans le port de Morlaix des quartiers de granites destinés à être taillés pour la bâtisse...[ils venaient] du bord de la mer, car plusieurs morceaux étaient recouverts par le... Balanus vulgaris...». Dans l'hospice de Saint-Pol-de-Léon, le granite de Callot se voit dans des portes et des fenêtres.

\section{Habitat}

À Morlaix, de nombreuses maisons ont employé naguère le granite de Callot comme pierre de taille. Les plus anciens édifices conservés paraissent remonter au XVI ${ }^{\mathrm{e}}$ siècle. Les constructions sont particulièrement fréquentes au XVIIe siècle et surtout au XVIII ${ }^{e}$ siècle et deviennent rares au XIX ${ }^{\mathrm{e}}$ siècle. Parfois une date précise peut être fournie : Rue Longue (1625), Rue Courte $(1666,1721)$, près de Saint-Mathieu (1754), Rue du Mur (1782), presbytère de Saint-Martin

(36) J. DARSEl. Histoire de Morlaix. Des origines à la Révolution, Rennes, 1942, 185 p.

(37) A. Guesdon. Mémoire sur la construction de l'Hôtel de Ville de Morlaix. Morlaix, A. Ledan.

(38) R. Pellen, 1986. Histoire de la manufacture des tabacs de Morlaix. Edit. du Dossen, Morlaix, $1838,110 \mathrm{p}$.

(39) Bigot de MOROGues (1809). Observations minéralogiques et géologiques sur les principales substances des départements du Morbihan, du Finistère et des Côtes-du-Nord. Journal des Mines, vol. 26, $\mathrm{n}^{\circ}$ 152, p. 81-111. «Les granites qu'on... emploie... [à Morlaix]... se ramassent au bord de la mer, ce qui est attesté par l'arrondissement de leurs angles et par les balanites qui les recouvrent... ils sont en masses considérables». La description des blocs de roches vus par cet auteur dans le port de Morlaix et, en particulier, la présence de «beaucoup de paillettes de mica noir» s'accordent très bien avec le granite de Callot. 


\section{Louis Chauris}

(1827). Les ensembles les plus intéressants sont situés Quai de Léon, Rue Longue, Rue Courte, Rue du Mur, Rue Haute, Rue A. de Guernisac, Grand Rue, sur les deux places au nord et au sud du Viaduc, place des Viarmes, Rue des Vignes, Rue de la Villeneuve... À Saint-Pol-de-Léon, les vieilles maisons en ce même granite sont assez fréquentes ; les dates s'échelonnent entre les XVI et XVIII' siècles (1532 - 1683 1773). À Roscoff, le granite de Callot est beaucoup plus rare (on pouvait faire ici largement appel aux granites locaux) ; on notera toutefois les beaux escaliers, parfaitement conservés, du vieux bâtiment de la station biologique. Plusieurs fermes (certaines sont d'anciens manoirs) ont utilisé aussi le granite de Callot, en particulier, comme il est normal, dans la commune de Carantec, à proximité des sites d'extraction. Leur nombre décrôit rapidement à Henvic et à Taulé, plus éloignés. À Kergadiou en Garlan, à Restedern en Plouigneau et à Coatilezec en Pleyber-Christ, l'emploi du granite de Callot est extrêmement restreint (fig.5).

\section{Utilisations diverses}

Le granite de Callot est entré naguère dans la confection des puits (Cosquer Ven en Carantec, Lesnoa en Henvic), de fontaines (sur le quai de Tréguier à Morlaix, «refaite et agrandie en 1716», en association avec le granite de l'île de Batz; au château de Keranroux en Ploujean), et surtout d'auges dont le façonnement révèle très bien l'aptitude de cette roche à la taille. L'inventaire des auges en granite de Callot est certainement loin d'être achevé : 4 ont été notées à Carantec, 2 à Plouézoc'h, 1 à Ploujean, 1 à Plouigneau et 1 à Saint-Martin-des-Champs. Certaines sont assez remarquables : Kermen et surtout Coat Morvan. La répartition des auges indique que dans le passé, les utilisateurs ne reculaient pas devant un certain transport quand il s'agissait d'une œuvre de qualité. Cette observation est générale dans le cas des auges en divers granites.

\section{Conclusions}

Malgré les réserves faites au début de la deuxième partie de ce mémoire (disparition d'édifices anciens, difficulté d'une enquête exhaustive, rareté des données d'archives), la comparaison des trois cartes de situation des constructions (fig.3 à 5), rapprochées des deux cartes des sites d'extraction (fig.1 et 2), permet de présenter un certain nombre de conclusions.

Dans son ensemble, le granite de Callot offre une utilisation essentiellement régionale. Sur les trois cartes, les divers types de constructions s'inscrivent dans une ellipse dont le grand axe et les limites varient peu, avec de très rares exceptions (cas de l'église de Saint-Jean-du-Doigt et des fermes de Kergadiou, Restedern et Coatilezec) ${ }^{(40)}$.

(40) Est-ce à dire que le beau granite de Callot n'a jamais été transporté à grande distance, comme ce fut le cas pour les granites de l'Aber-Ildut (jusqu'en Angleterre) et de l'Ile Grande 
La répartition des édifices en granite de Callot est essentiellement sous la dépendance du transport par voie d'eau, à savoir la rade et la rivière de Morlaix (ou Dossen), plus accessoirement la Penzé. Ces deux rias qui pénètrent profondément à l'intérieur des terres ont souvent permis l'acheminement du granite sur les lieux mêmes de son utilisation ou, tout au moins, à proximité. L'église de Guiclan, située à près de $6 \mathrm{~km}$ du port de Penzé, représente déjà un cas-limite. Bien que les documents écrits fassent défaut, il est probable que le granite de Callot, employé localement dans l'église de Saint-Jean-du-Doigt, a été également transporté par mer. Sans la possibilité d'acheminement maritime, cette roche, malgré ses grandes qualités, n'aurait guère dépassé les limites des communes de Carantec et de Saint-Pol-de-Léon.

Au total, dans l'état actuel des recherches, le granite de Callot a été observé dans 18 communes : outre les deux communes précitées, Henvic, Taulé, Locquénole, Morlaix, Ploujean, Plouézoc'h, Saint-Martin-des-Champs, Saint-Sève, SaintJean-du-Doigt, Plouigneau, Garlan, Guiclan, Saint-Thégonnec, Pleyber-Christ, Plouénan et Roscoff. Morlaix, alors cité commerçante prospère et, dans une moindre mesure, Saint-Pol-de-Léon, siège d'un évêché, sont, de loin, les plus gros demandeurs. Roscoff utilisait surtout le beau granite de l'île de Batz toute proche. La rareté relative du granite de Callot à Carantec s'explique essentiellement par la pauvreté de cette ancienne trève de la paroisse de Taulé : il fallait, à l'évidence, disposer d'une certaine aisance pour employer la pierre de taille : aussi peut-on comprendre que cette roche ait été ici surtout utilisée - mis à part l'église paroissiale et la chapelle N-D de Callot - dans les constructions nobles (Cosquer Ven, Kerhallic, Kerangoaguet...). Les anciennes constructions ordinaires ont, le plus souvent, recherché des moellons de gneiss locaux ou de granite de Carantec, d'extraction et de façonnement aisés.

L'évolution dans l'emploi du granite de Callot au cours des temps est contrôlée par la concurrence avec d'autres roches régionales de qualité. À notre connaissance, ce granite ne semble pas avoir été utilisé dans les mégalithes, ni dans les stèles de l'Age du Fer - ou du moins, aucun vestige ne nous est parvenu. Dans les premiers édifices en pierre de taille de la région morlaisienne, le granite de Ploujean, extrait du Menez et de Kerscoff, a été largement utilisé (partie romane de l'église

(jusqu'en Belgique) ? L'analyse d'un document ancien suggère qu'il n'en est rien. En 1788, 11 navires, jaugeant au total 1637 tx. ont transporté des «pierres» à partir du port de Morlaix, à destination de l'étranger (Hollande en général et le port d'Amsterdam en particulier) (donnée citée par J.C MORAUD : Quelques aspects du port de Morlaix et de son trafic à la veille de la révolution 1781-1789. Mém. Maîtrise, Univ. Paris I, Sorbonne,1981, 200 p.), soit plus de deux fois plus en une année aux exportations qu'en 9 ans (1781-1789) aux importations. Comme à l'évidence du fait du port d'embarquement - il ne peut être question de kersanton, ni des granites de l'Aberlldut ou de l'lle Grande, tout laisse à penser, selon nous, qu'il pourrait s'agir du granite de l'île Callot - dont c'était alors (XVIII ${ }^{e}$ siècle) la période de grande extraction - à destination d'un pays totalement dépourvu de roches granitiques. Ainsi, pendant un laps de temps peut-être assez bref, le granite de Callot aurait-il eu aussi une diffusion internationale ? 
de Ploujean, $\mathrm{XI}^{\mathrm{e}}$ siècle ; partie gothique du même édifice, XIVe siècle...) ; l'emploi de ce granite clair, de taille aisée, a été très important jusqu'au XVII ${ }^{e}$ siècle inclus ${ }^{(4)}$. Mais dès la fin du XVe siècle, il était concurrencé par le granite de l'île de Batz et, peu à peu, par le granite de l'île Callot. L'utilisation de ce dernier granite est maximale au XVIII ${ }^{\mathrm{e}}$ siècle et décroît au XIX $\mathrm{X}^{\mathrm{e}}$ siècle ; au cours de cette dernière période, les granites de l'Ile Grande, déjà concurrentiels dès le XVII siècle, allaient «étouffer» le granite de Callot, avant d'être, à leur tour, dans la région de Morlaix, supplantés vers la fin du $\mathrm{XIX}^{\mathrm{e}}$ siècle et surtout au $\mathrm{XX}^{\mathrm{e}}$ siècle, par le granite du Huelgoat.

Est-ce à dire que le granite de Callot n'a plus aucun avenir ? Carantec et d'une manière plus générale toute la baie de Morlaix est résolument tournée vers le tourisme et il n'est évidemment pas question de transformer l'île paradisiaque de Callot en un chantier d'extraction. Toutefois, pour la restauration de nombreux édifices de la région morlaisienne, il serait peut-être possible d'envisager, dans un secteur de l'île, une petite exploitation artisanale qui suffirait aux besoins. Une telle réalisation, sans dommage pour l'environnement, serait, à notre sens, l'expression d'une compréhension éclairée pour la sauvegarde du matrimoine architectural dans le Pays de Morlaix.

(41) L. Chauris. «Une belle pierre oubliee : le granite albitique de Ploujean près Morlaix (Finistère)». Revue archéol. de l'Ouest. 1993, 10, p.141-155. 
L'extraction du granite rose de l'île Callot...

\begin{tabular}{|c|c|c|c|}
\hline 1 & 2 & 3 & 4 \\
\hline $11-10-5$ & $9,5-6,5-7$ & $18-13-4$ & $12,5-6,5-4$ \\
\hline $11-10-5$ & $10,5-5,5-9$ & $17-12-3,5$ & $12,5-6-5,5$ \\
\hline $14-9-11$ & $11-5-7$ & $14-12-3,5$ & $12,5-6,5-5$ \\
\hline $13-8-6$ & $10,5-5,5-8$ & $14-11-3,5$ & $12-5,5-3$ \\
\hline $14-10-4,5$ & $10,5-5-8,5$ & $13,5-10-3$ & $13-6-4$ \\
\hline $13-7-5,5$ & $11-6-10$ & $15-10-1,5$ & $12-6,5-5,5$ \\
\hline $12-7-9$ & $11-6-10,5$ & $11-11-2$ & $13-6,5$ \\
\hline . . . & $11,5-6-7,5$ & $13-12-1$ & $12,5-6,2-4,5$ \\
\hline $13-8-8$ & $12,5-6-6,5$ & $14-11-2$ & $\mathrm{~L} / \mathrm{I}=2$ \\
\hline $12-8-7$ & $11-5,5-7,5$ & $13-9-4$ & \\
\hline $13-9-4,5$ & $10,5-5,5-7$ & $13-7-4$ & 5 \\
\hline $13-9-6,5$ & $11,5-5-5,5$ & $12-8-4$ & \\
\hline $13-9-6,5$ & $10,5-5-5$ & $14-9$ & L I \\
\hline $11,5-6,5-9$ & $11,5-5,5-8,5$ & & $14-7$ \\
\hline $12-9-9$ & $11,5-6,5-8$ & & $16-8$ \\
\hline $11-9-9$ & $11,5-6$ & & $17-11$ \\
\hline $11-11-6,5$ & & & $16-12$ \\
\hline $13-11,5$ & & & $16-13$ \\
\hline & & & $16-12$ \\
\hline $12,4-8,9-7$ & $11-5,6-7,7$ & $14-10,4-3$ & $15,8-10,5$ \\
\hline $\mathrm{L} / \mathrm{I}=1,4$ & $\mathrm{~L} / \mathrm{I}=1,9$ & $\mathrm{~L} / \mathrm{I}=1,3$ & $\mathrm{~L} / \mathrm{I}=1,5$ \\
\hline
\end{tabular}

Tableau 1 - Successivement longueur, largeur et espacement des trous pour les coins dans le granite de l'île Callot.

1 et 2 - Entre Vougeo et Porz an Ilis.

3 - Près de Porz Moualc'h.

4 et 5 - Run Lann. (. . . = légère interrruption). 


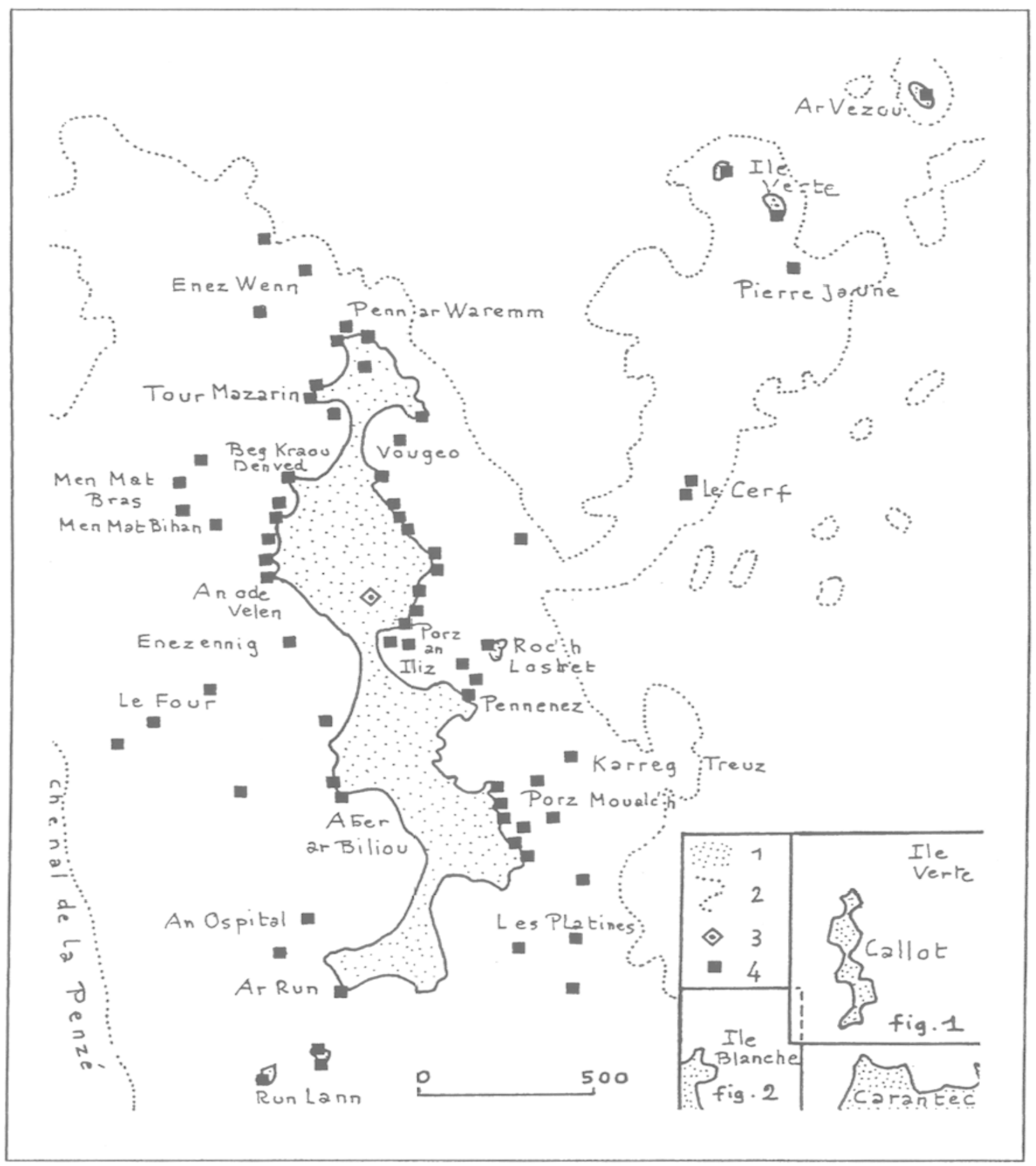

Fig. 1 - Localisation des anciennes perrières d l'île Callot et autour de l'île Verte

1 - Zone toujours émergée.

2 - Limite des plus basses mers.

3 - Chapelle N-D. de Callot.

4 - Perrière abandonnée. 
L'extraction du granite rose de l'île Callot...

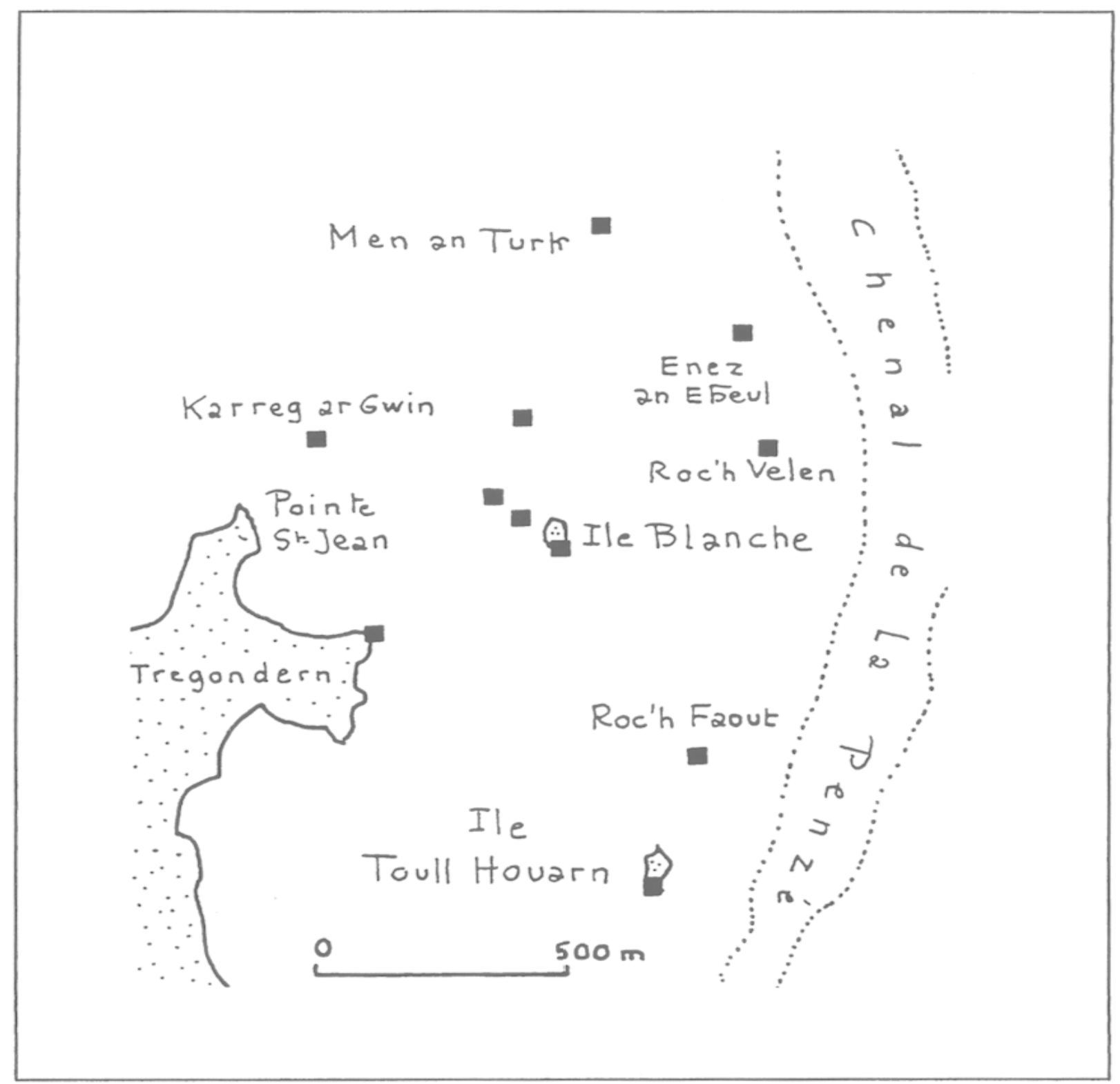

Fig. 2 - Localisation des anciennes perrières autour de l'Ile Blanche

1 -Zone toujours émergée.

2 - Limite des plus basses mers.

3 - Chapelle N-D. de Callot.

4 - Perrière abandonnée. 


\section{Louis Chauris}

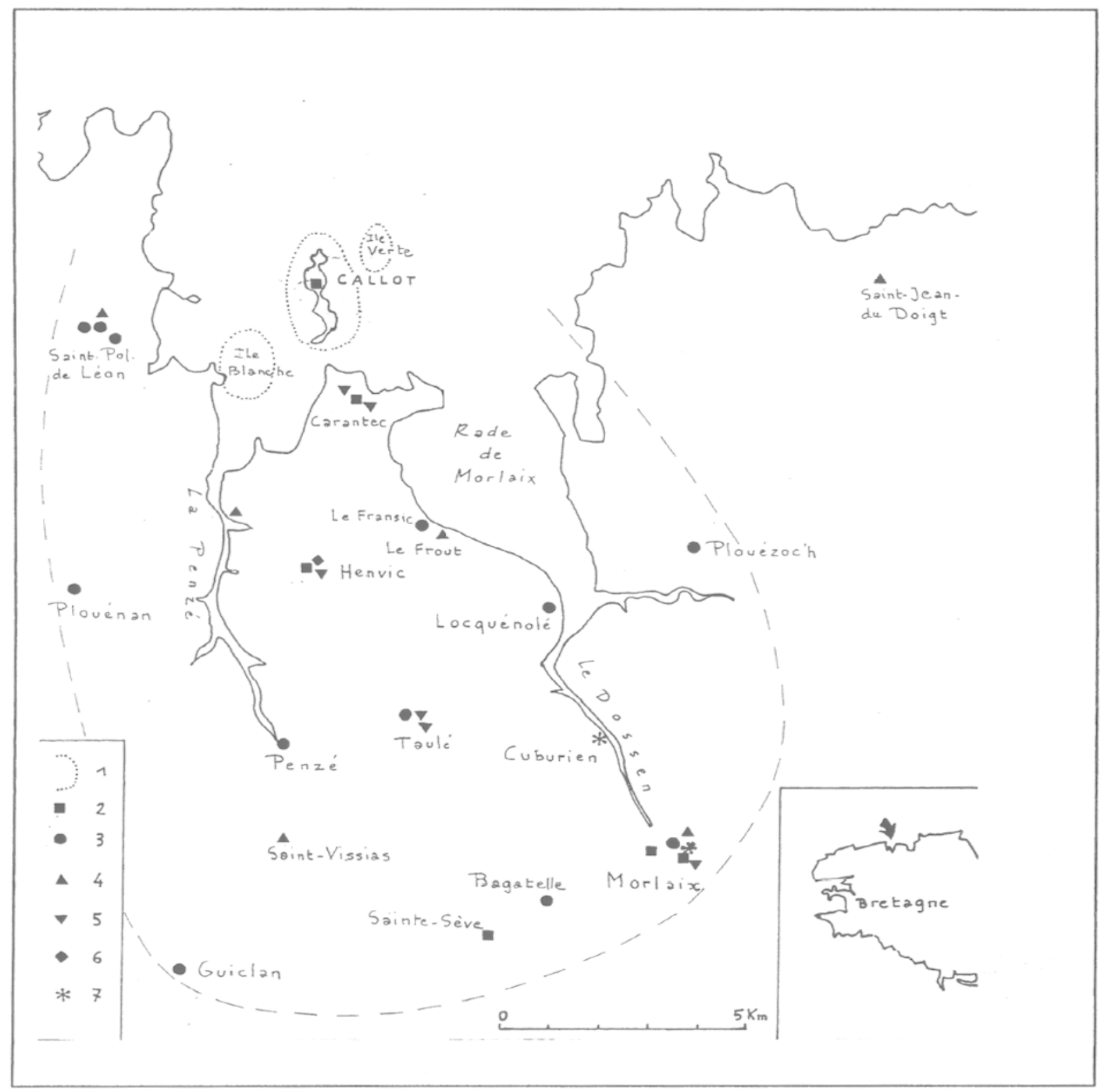

Fig 3 - Edifices religieux (sensu lato) ayant utilisé le granite de Callot

1-Zone d'extraction du granite (schématisée).

2 a 4 - Proportion relative en granite de Callot :

2 - Importante.

3 - Restreinte.

4 - Rare.

5 - Calvaire.

6-Dalle tumulaire.

7 - Bâtiment conventuel. 
L'extraction du granite rose de l'île Callot...

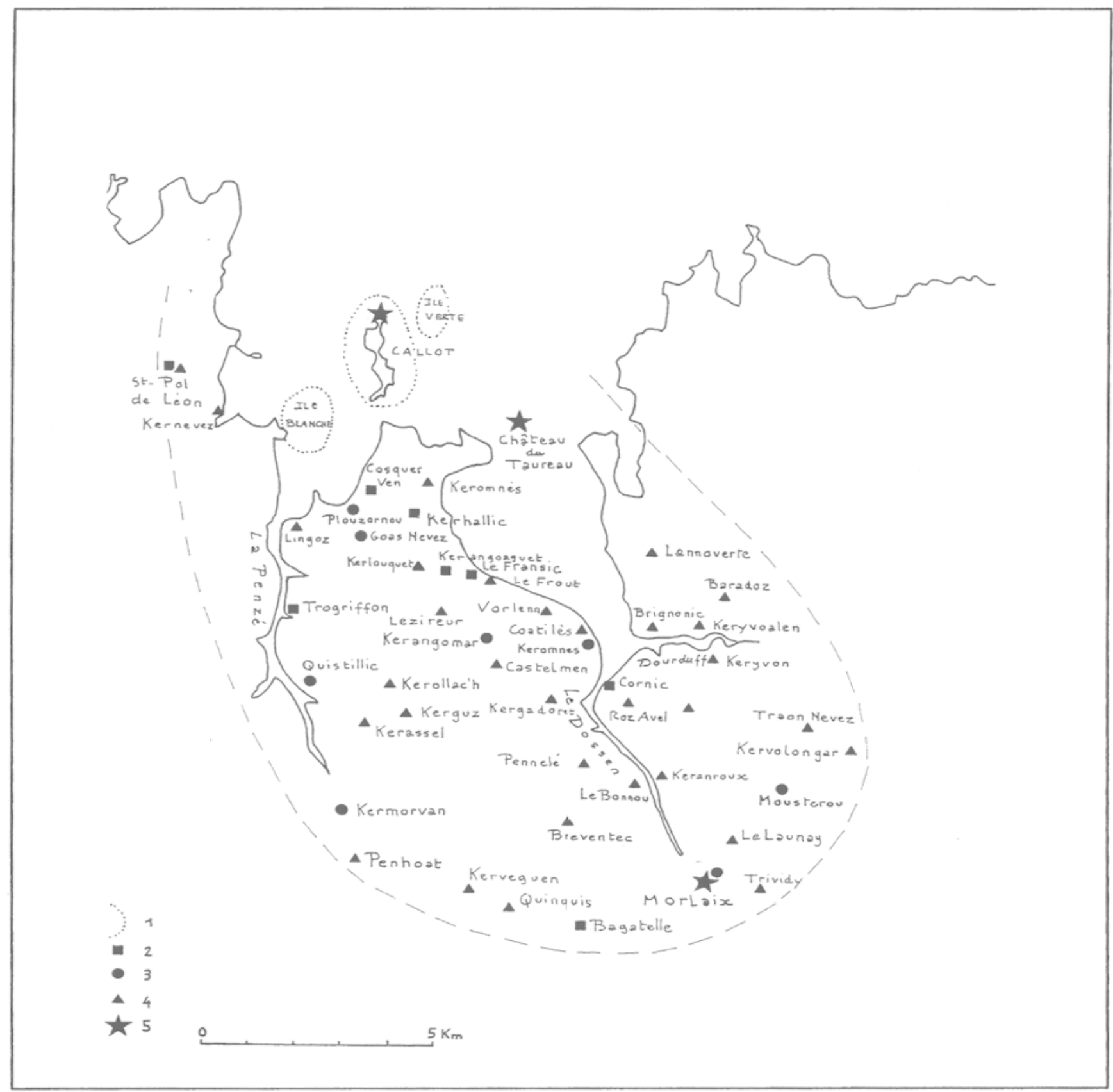

Fig. 4 - Châteaux, manoirs et ouvrages défensifs ayant employé le granite de Callot

1 - Zone d'extraction du granite (schématisée).

2 à 4 - Proportion relative en granite de Callot :

2 - Importante.

3 - Restreinte.

4 - Rare.

5 - Ouvrage défensif. 


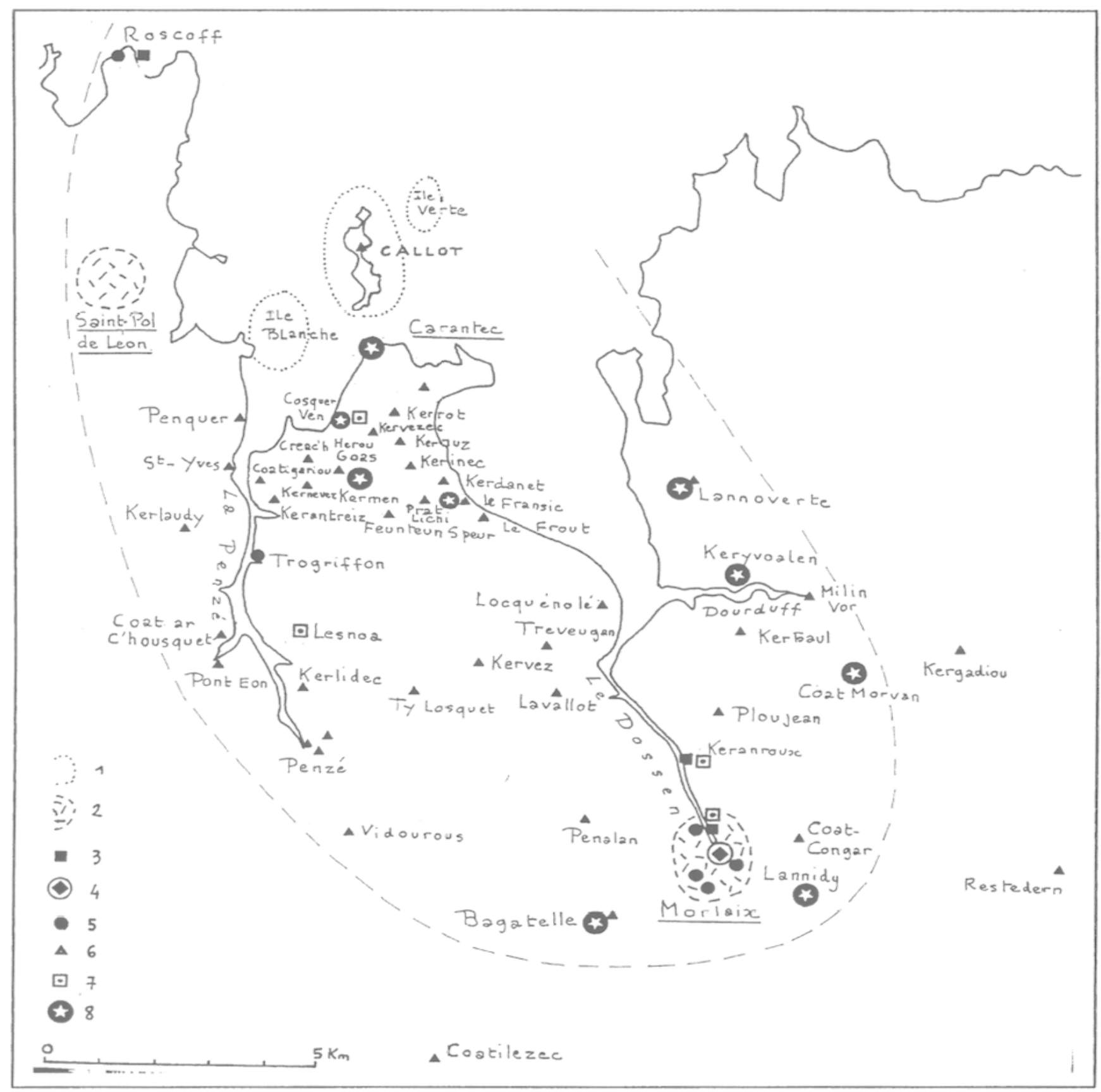

Fig. 5 - Constructions diverses ayant utilisé le granite de Callot

1 - Zone d'extraction (schématisée).

2 - Ville avec large emploi du granite de Callot dans l'habitat.

3 - Ouvrage portuaire.

4 - Ouvrage ferroviaire.

5 - Édifice divers.

6 - Habitat rural.

7 - Puits ou fontaine.

8 - Auge. 
L'extraction du granite rose de l'île Callot...

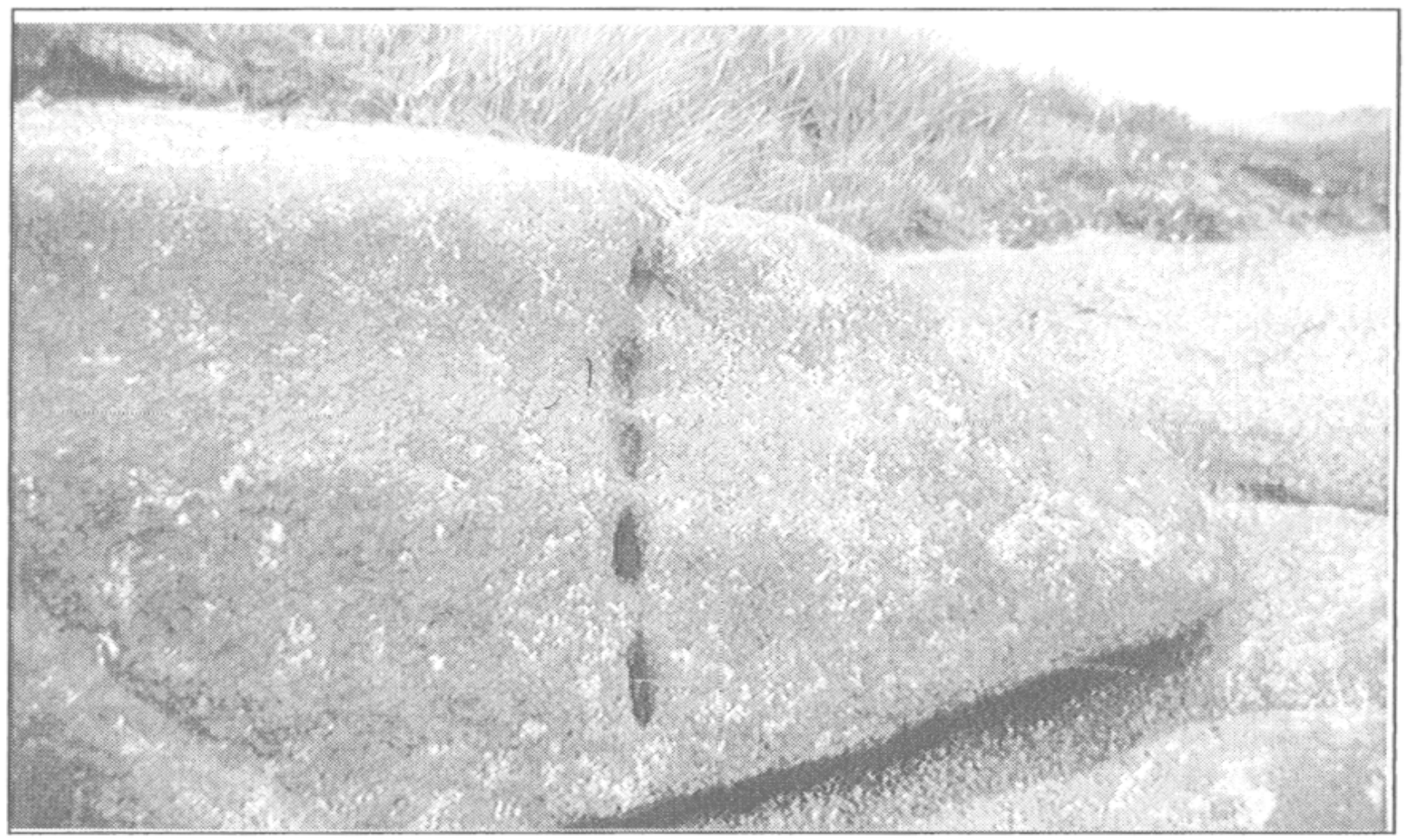

Trous pour l'emplacement des coins à l'île Blanche.

Château du Taureau.

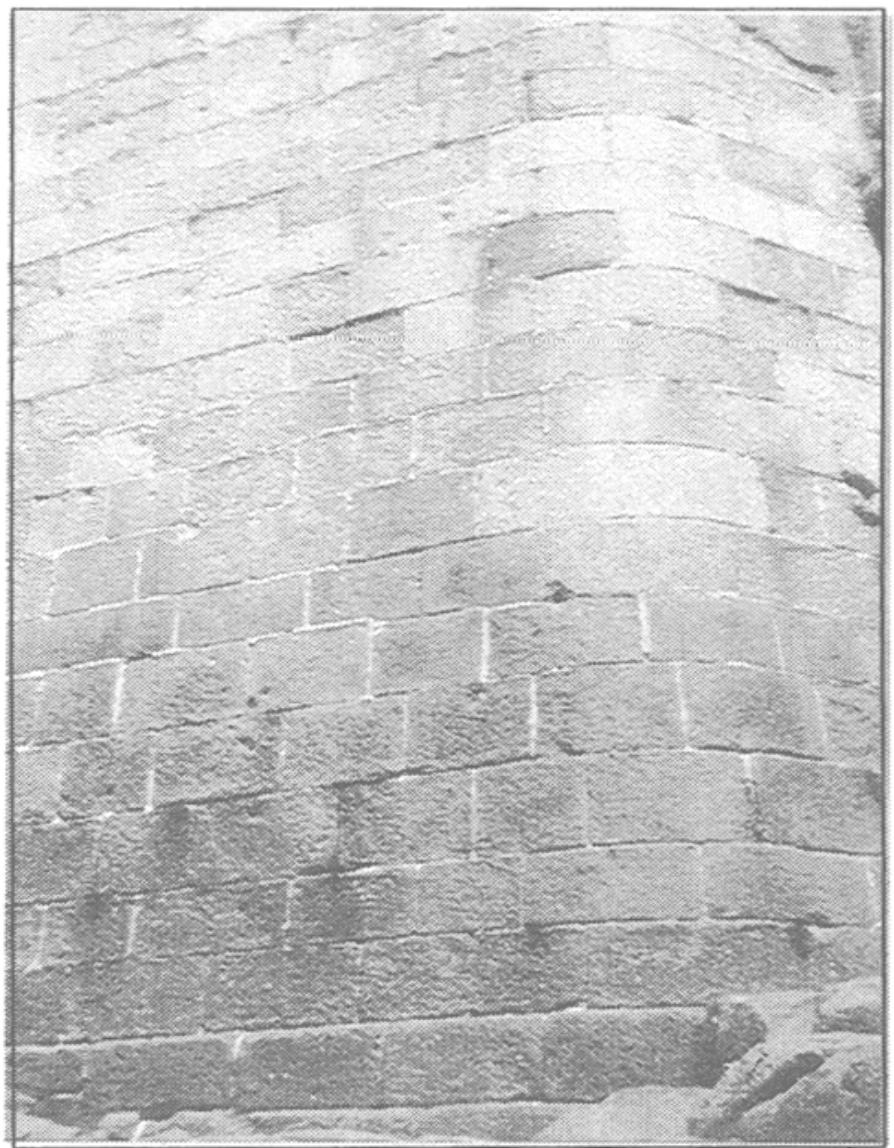

Granite de Callot, en grand appareil. 


\section{Louis Chauris}

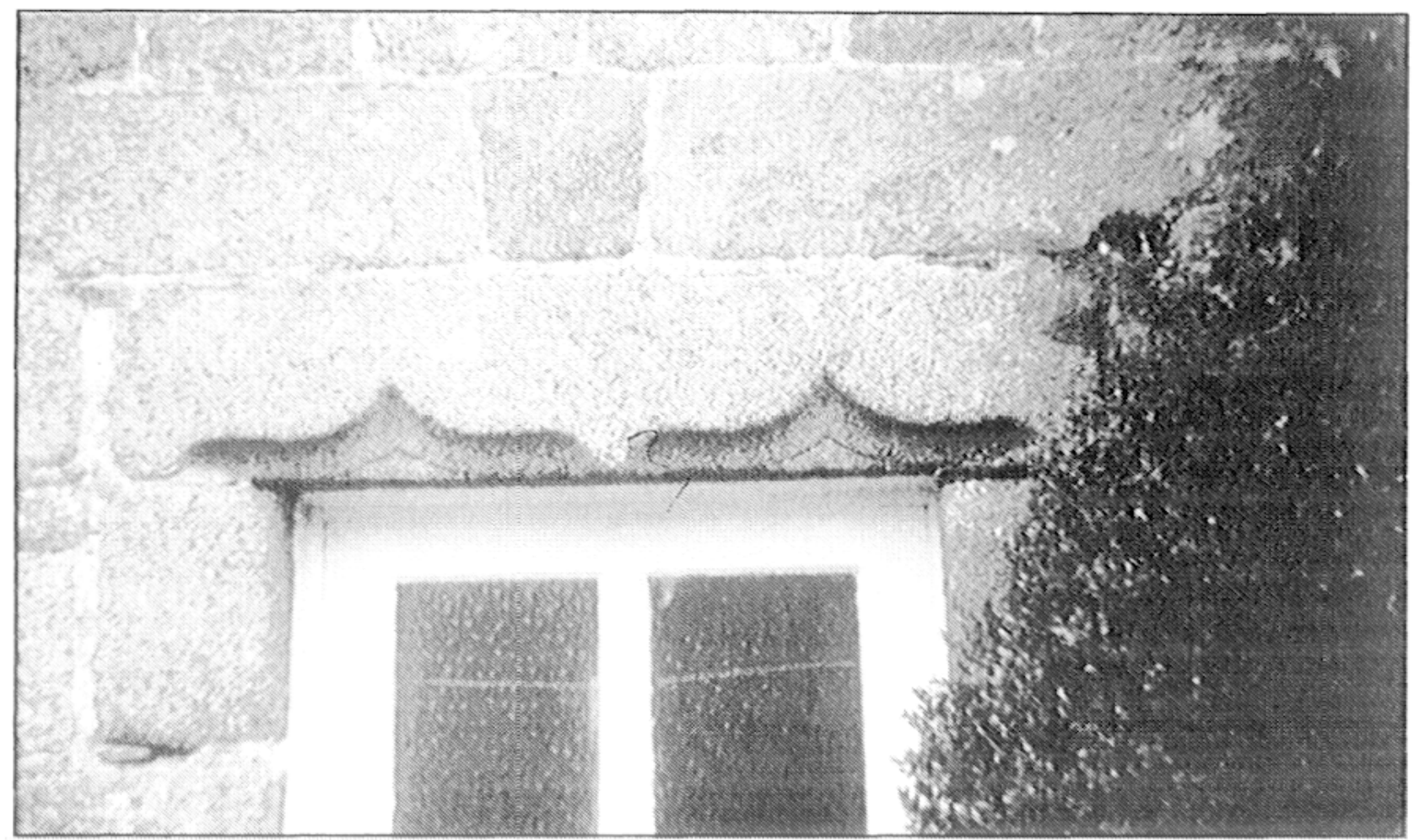

Linteau à double accolade, en granite de Callot. Manoir de Cosquer Ven en Carantec.

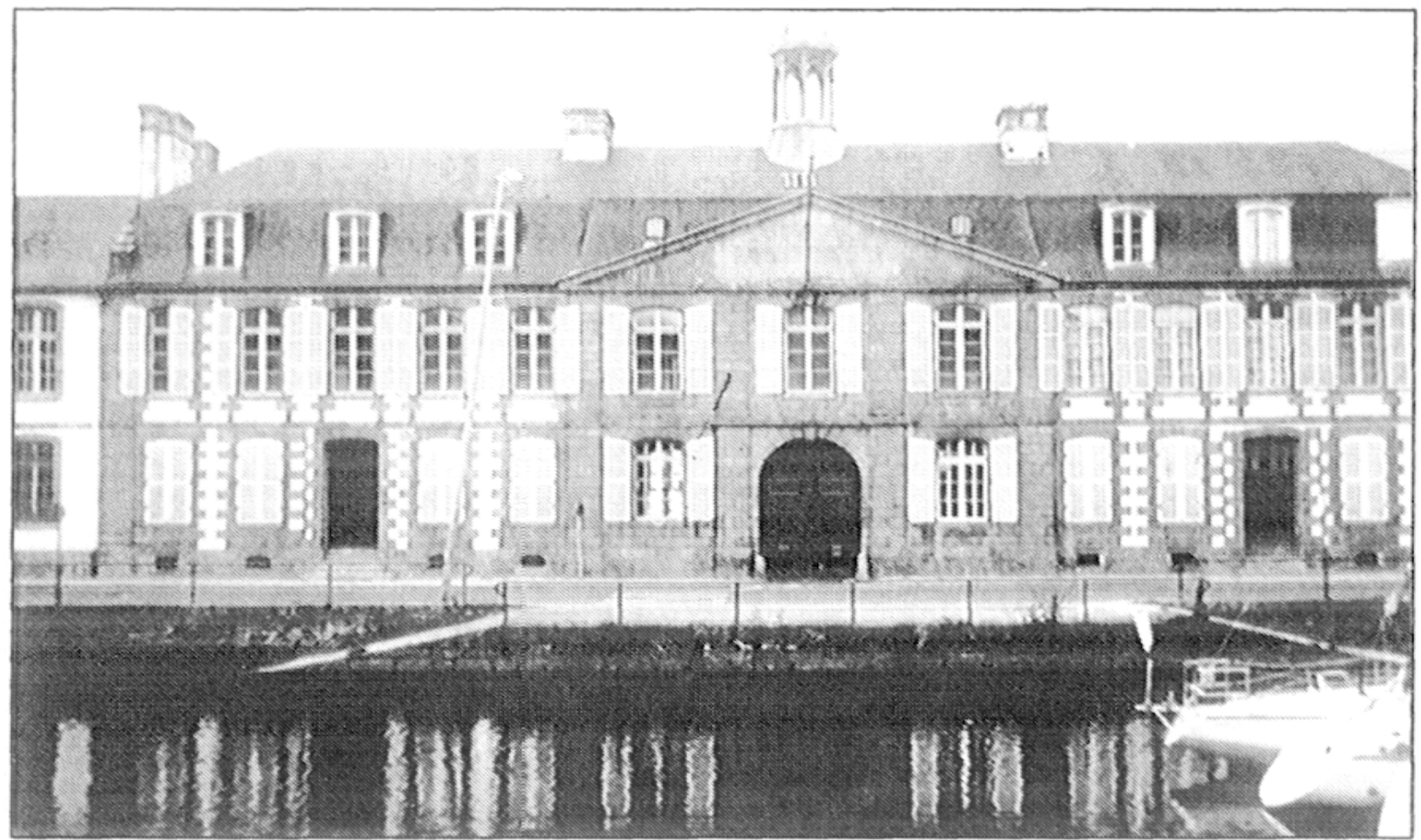

Manufacture de tabacs à Morlaix, en granite de Callot (1736-1740). 


\section{L'extraction du granite rose de l'île Callot...}

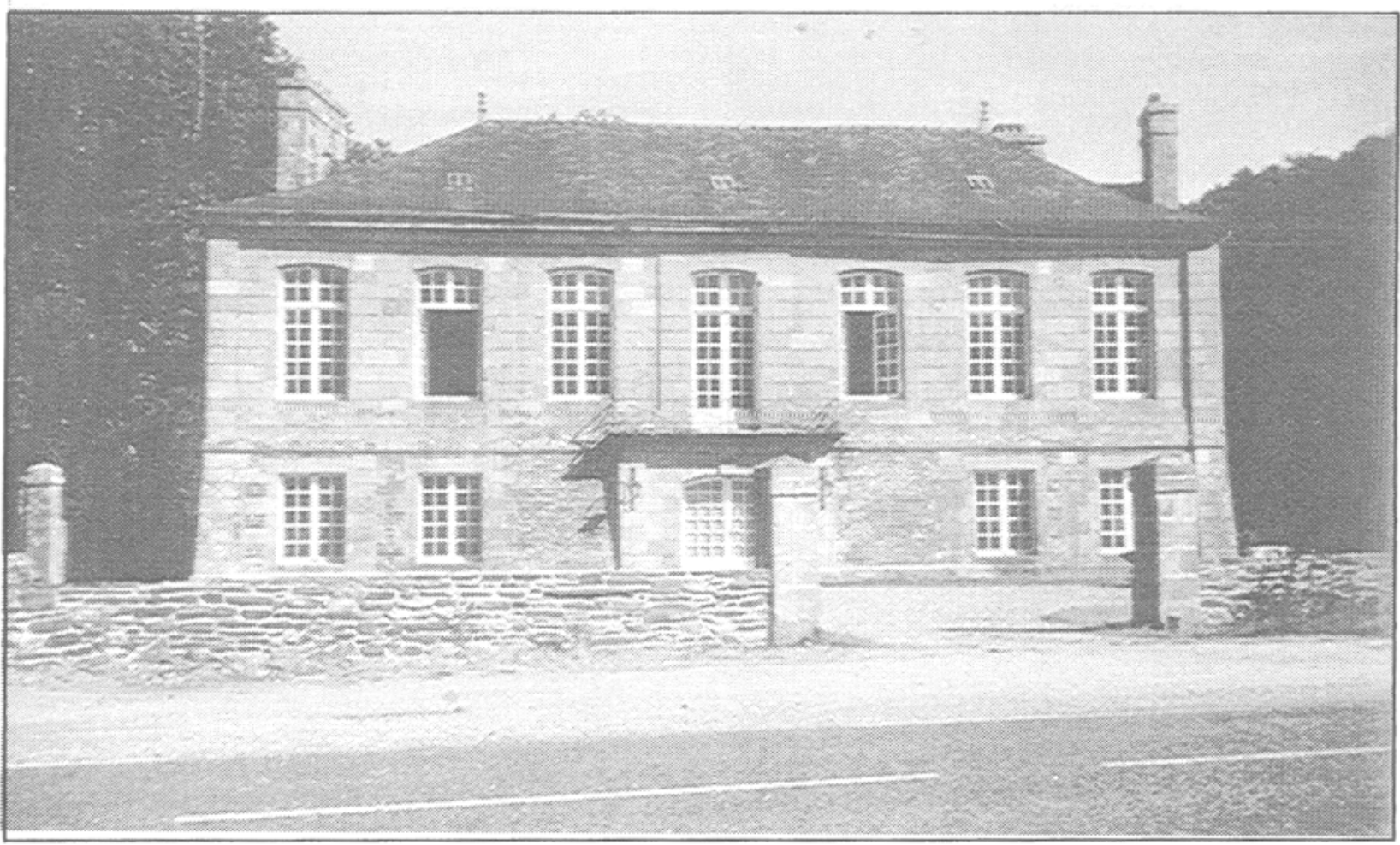

Maison dite de «Cornic» en Ploujean, en granite de Callot.

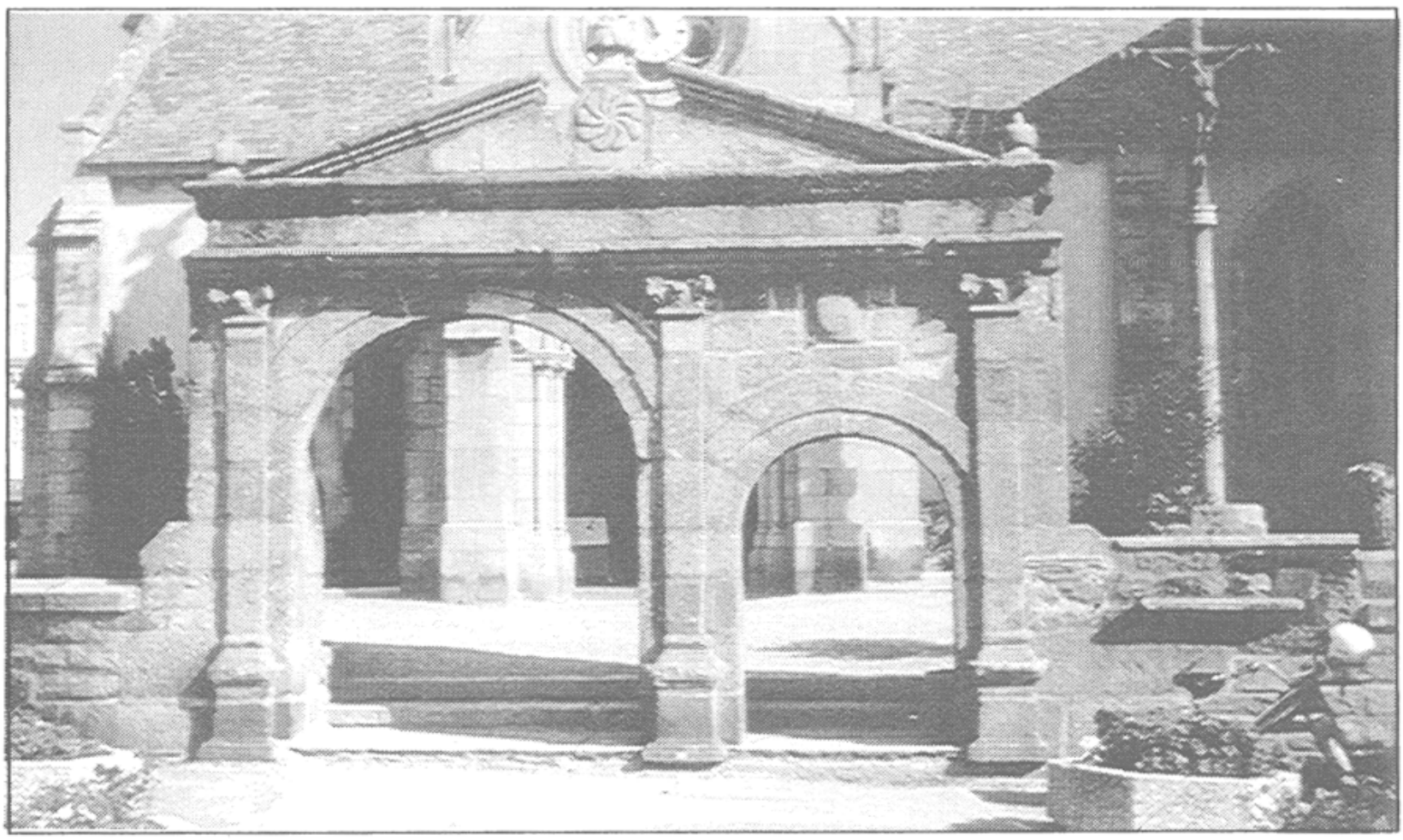

Ancien portail du manoir de Kerangoaguet, à présent devant l'église de Carantec. 


\section{Louis Chauris}

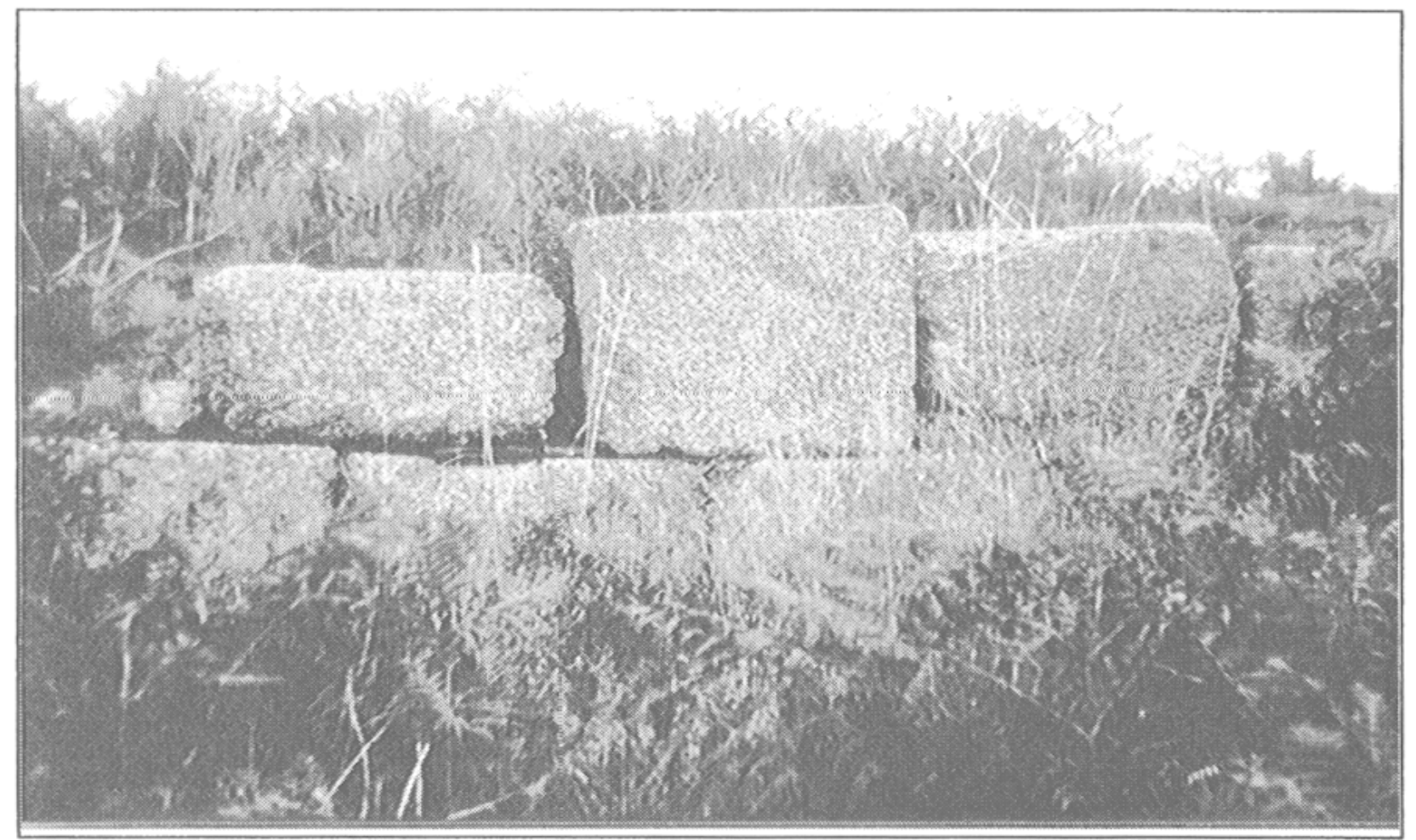

Ruines de l'ancien fort de Callot, en granite de l'île. 